\title{
ALMOST PERIODIC HOMOGENIZATION OF A GENERALIZED LADYZHENSKAYA MODEL FOR INCOMPRESSIBLE VISCOUS FLOW
}

\author{
HERMANN DOUANLA AND JEAN LOUIS WOUKENG
}

\begin{abstract}
The paper deals with the existence and almost periodic homogenization of some model of generalized Navier-Stokes equations. We first establish an existence result for non-stationary Ladyzhenskaya equations with a given non constant density and an external force depending nonlinearly on the velocity. Next, the density of the fluid being non constant, we combine some compactness arguments with the sigma-convergence method to study the asymptotic behavior of the velocity field.
\end{abstract}

\section{INTRODUCTION}

The Navier-Stokes equations model the motion of Newtonian fluids. In order to understand the phenomenon of turbulence related to the motion of a fluid, several mathematical models have been developed and studied over the years. We refer to, e.g. [2, 10, 18, 20, 22, just to cite a few.

To investigate the turbulence in non-Newtonian fluid, we consider a model close to the Ladyzhenskaya one 20. In this model, the viscosity and the density are non constant. To be more precise, let $\varepsilon>0$ be a small parameter representing the scale of the inhomogeneities. The equation of the motion reads

(1.1)

$\rho^{\varepsilon} \frac{\partial \boldsymbol{u}_{\varepsilon}}{\partial t}-\operatorname{div}\left(a^{\varepsilon} \nabla \boldsymbol{u}_{\varepsilon}+b^{\varepsilon}\left|\nabla \boldsymbol{u}_{\varepsilon}\right|^{p-2} \nabla \boldsymbol{u}_{\varepsilon}\right)+\left(\boldsymbol{u}_{\varepsilon} \cdot \nabla\right) \boldsymbol{u}_{\varepsilon}+\nabla q_{\varepsilon}=\rho^{\varepsilon} f^{\varepsilon}\left(\cdot, \boldsymbol{u}_{\varepsilon}\right)$ in $Q_{T}$

where $\rho$ is the non constant positive known density which is bounded from above and from below away from zero, $\boldsymbol{u}_{\varepsilon}$ and $q_{\varepsilon}$ are the unknown velocity and pressure, respectively. The viscosities $a=\left(a_{i j}\right)_{1 \leq i, j \leq N}$ and $b$ both depend on the spatial and time variables. The matrix $a$ is coercive whereas the function $b$ is positive, bounded from above and from below away from zero. The density and the viscosities are scaled as follows: $\rho^{\varepsilon}(x)=\rho\left(\frac{x}{\varepsilon}\right), a_{i j}^{\varepsilon}(x, t)=a_{i j}\left(\frac{x}{\varepsilon}, \frac{t}{\varepsilon^{2}}\right)$ and $b^{\varepsilon}(x, t)=b\left(\frac{x}{\varepsilon}, \frac{t}{\varepsilon^{2}}\right)$ whereas the external force $f^{\varepsilon}\left(\cdot, \boldsymbol{u}_{\varepsilon}\right)$ which is a Lipschitz function of the velocity $\boldsymbol{u}_{\varepsilon}$ is scaled as follows $f^{\varepsilon}(\cdot, r)(t)=f\left(\frac{t}{\varepsilon^{2}}, r\right)$ for $(x, t) \in Q_{T}$ and $r \in \mathbb{R}^{N}$. The problem is stated in details in the beginning of section 2 .

The equation (1.1) models various types of motion of non-Newtonian fluids. We cite a few examples. If $a=\left(\nu_{0} \delta_{i j}\right)_{1 \leq i, j \leq N}\left(\delta_{i j}\right.$ the Kronecker delta) and $b=\nu_{1}$ where $\nu_{0}$ and $\nu_{1}$ are positive constants, then we get the Ladyzhenskaya equations. In that case, the analysis conducted in [18 reveals that one may either let $\nu_{1} \rightarrow 0$ (and get the usual Navier-Stokes equations) or let $\nu_{0} \rightarrow 0$, and hence we are led to the power-law fluids equations. In particular when $p=3$, we get the Smagorinski's

2000 Mathematics Subject Classification. 35B40, 46J10, 76D05.

Key words and phrases. Homogenization, Almost periodic, Navier-Stokes equations. 
model of turbulence 36] with $\nu_{0}$ being the molecular viscosity and $\nu_{1}$ the turbulent viscosity. Another model included in (1.1) is the equation of incompressible bipolar fluids [2].

There are several works dealing with the Navier-Stokes equations with external force depending on the velocity. These types of equations are commonly known as the generalized Navier-Stokes equations. This work is different from the previous ones in the sense that it combines both a non-constant density, the non-Newtonian fluid effect, and the external force depending on the velocity. It should be noted that if in equation (1.1) we replace the gradient $\nabla \boldsymbol{u}$ by its symmetric part $\frac{1}{2}\left(\nabla \boldsymbol{u}+\nabla^{T} \boldsymbol{u}\right)$, then thanks to the Korn's inequality, the mathematical analysis does not change, although the model becomes in this case, physical.

Started in the 70's the mathematical theory of Homogenization is nowadays divided into two major components: the individual homogenization theory (also known as the deterministic homogenization theory) and the random homogenization theory (also known as the stochastic homogenization theory). In this paper we are concerned with a special case of individual homogenization theory, namely the almost periodic one. More precisely, we assume throughout the paper that the density $\rho$, the viscosities $a$ and $b$ and the source term $f$ are almost periodic functions as specified in the beginning of Section 4. It should be noted that we could consider a more general deterministic setting with coefficients satisfying general deterministic assumptions covering a large set of concrete behaviors (see e.g., [13, 23, 24]) such as the periodic one, the almost periodic one, the convergence at infinity and many more besides. We only deal with almost periodic homogenization for the sake of simplicity. Our results carry over mutatis mutandis to the general deterministic setting. Concerning the homogenization of (1.1), it should also be stressed that this is the first time that such an analysis is conducted beyond the periodic setting.

The goal of this paper is twofold. We first establish an existence result for (1.1) and then perform the homogenization process for (1.1) in the almost periodic setting. The space dimension is either 2 or 3 . The only place where the analysis changes depending on the dimension is the proof of the inequality (2.8) which is very useful in the proofs of the existence result, theorem 1 and the compactness result, Proposition 1. We stress that the existence result Theorem 1 is not solving the millennium problem of the existence and smoothness of the Navier-Stokes equations as stated by Fefferman [17] since we only consider weak solutions here with no regularity result.

The paper is organized as follows. Section 2 deals with the complete statement of the problem, the proof of the existence result and some a priori estimates. In Section 3 we gather some necessary tools about the $\Sigma$-convergence method (which is just the appropriate generalization of the well-known two-scale convergence method) in the algebra of continuous almost periodic functions. Finally in Section 4, we state and prove the homogenization result.

Throughout Section 3, vector spaces are assumed to be complex vector spaces, and scalar functions are assumed to take complex values. We shall always assume that the numerical space $\mathbb{R}^{m}$ (integer $m \geq 1$ ) and its open sets are each equipped with the Lebesgue measure $d x=d x_{1} \ldots d x_{m}$. 


\section{Statement of the Problem: existence Result and A PRIORi estimates}

2.1. Problem setting: existence result. We consider $N$-dimensional problem, $N=2,3$. In what follows, all the function spaces are real-valued spaces and scalar functions assume real values.

Let $1+\frac{2 N}{N+2} \leq p<\infty$, and $T>0$ a real number. Let $Q_{T}=Q \times(0, T)$ where $Q$ is a bounded smooth domain in $\mathbb{R}^{N}$. We consider the following well-known spaces [22, 38]: $\mathcal{V}=\left\{\varphi \in \mathcal{C}_{0}^{\infty}(Q)^{N}: \operatorname{div} \varphi=0\right\} ; \mathbb{V}=$ closure of $\mathcal{V}$ in $W^{1, p}(Q)^{N}$; $\mathbb{H}=$ closure of $\mathcal{V}$ in $L^{2}(Q)^{N}$. In view of the smoothness of $Q$, it is known that $\mathbb{V}=\left\{\boldsymbol{u} \in W_{0}^{1, p}(Q)^{N}: \operatorname{div} \boldsymbol{u}=0\right\}$ and $\mathbb{H}=\left\{\boldsymbol{u} \in L^{2}(Q)^{N}: \operatorname{div} \boldsymbol{u}=0\right.$ and $\left.\left.\boldsymbol{u}\right|_{\partial Q} \cdot n=0\right\}$, where $\left.\boldsymbol{u}\right|_{\partial Q}$ denotes the trace of $\boldsymbol{u}$ on $\partial Q$ and $n$ is the outward unit vector normal to $\partial Q$. The space $\mathbb{V}$ is hence endowed with the gradient norm and $\mathbb{H}$ with the $L^{2}$-norm. For the sake of simplicity, we denote the respective norms of $\mathbb{V}$ and $\mathbb{H}$ by $\|\cdot\|$ and $|\cdot|$. We denote by $($,$) the inner product in \mathbb{H}$, as well as the scalar product in $\mathbb{R}^{N}$. The associated Euclidean norm in $\mathbb{R}^{N}$ is also denoted by $|\cdot|$. All duality pairing will be denoted by $\langle$,$\rangle without any specification of the spaces$ involved.

With all this in mind, we shall consider the functions $a, b, \rho$ and $f$ constrained as follows.

(A1) The matrix $a=\left(a_{i j}\right)_{1 \leq i, j \leq N} \in L^{\infty}\left(\mathbb{R}^{N+1}\right)^{N \times N}$ satisfies $a_{i j}=a_{j i}$ and

$$
(a(y, \tau) \lambda) \cdot \lambda \geq \nu_{0}|\lambda|^{2} \text { for all } \lambda \in \mathbb{R}^{N} \text { and a.e. }(y, \tau) \in \mathbb{R}^{N+1}
$$

for some positive $\nu_{0}$.

(A2) The function $b \in L^{\infty}\left(\mathbb{R}^{N+1}\right)$ is such that $\nu_{1} \leq b \leq \nu_{2}$ for some positive $\nu_{1}$ and $\nu_{2}$.

(A3) The density function $\rho$ belongs to $\in L^{\infty}\left(\mathbb{R}^{N}\right)$ and satisfies $\Lambda^{-1} \leq \rho(y) \leq \Lambda$ a.e. $y \in \mathbb{R}^{N}$, for some positive $\Lambda$.

(A4) The mapping $f: \mathbb{R} \times \mathbb{R}^{N} \rightarrow \mathbb{R}^{N},(t, r) \mapsto f(t, r)$ is continuous with the following properties:

(i) $f$ maps continuously $\mathbb{R} \times \mathbb{H}$ into $\mathbb{H}(f(\tau, u) \in \mathbb{H}$ for any $u \in \mathbb{H})$

(ii) There is a positive constant $k$ such that

$$
\begin{aligned}
& |f(\tau, 0)| \leq k \text { for all } \tau \in \mathbb{R} \\
& \left|f\left(\tau, r_{1}\right)-f\left(\tau, r_{2}\right)\right| \leq k\left|r_{1}-r_{2}\right| \text { for all } r_{1}, r_{2} \in \mathbb{R}^{N} \text { and } \tau \in \mathbb{R} .
\end{aligned}
$$

We deduce from [part (ii) of] (A4) the existence of a positive constant $c$ such that $|f(\tau, r)| \leq c(1+|r|)$ for any $(\tau, r) \in \mathbb{R} \times \mathbb{R}^{N}$. The scaled functions $\rho^{\varepsilon} \in L^{\infty}(Q)$, $a^{\varepsilon}=\left(a_{i j}^{\varepsilon}\right)_{1 \leq i, j \leq N} \in L^{\infty}\left(Q_{T}\right)^{N \times N}, f^{\varepsilon}(\cdot, r) \in \mathcal{C}(0, T)$ (for any $r \in \mathbb{R}^{N}$ ) and $b^{\varepsilon} \in$ $L^{\infty}\left(Q_{T}\right)$ are defined as follows:

$$
\begin{aligned}
\rho^{\varepsilon}(x) & =\rho\left(\frac{x}{\varepsilon}\right), a_{i j}^{\varepsilon}(x, t)=a_{i j}\left(\frac{x}{\varepsilon}, \frac{t}{\varepsilon^{2}}\right), f^{\varepsilon}(\cdot, r)(t)=f\left(\frac{t}{\varepsilon^{2}}, r\right) \text { and } \\
b^{\varepsilon}(x, t) & =b\left(\frac{x}{\varepsilon}, \frac{t}{\varepsilon^{2}}\right) \text { for }(x, t) \in Q_{T} .
\end{aligned}
$$

Let $\varepsilon>0$ be a small parameter. We will make use of the following notation

$$
(\boldsymbol{u}, \boldsymbol{v})_{\varepsilon}=\int_{Q} \rho^{\varepsilon}(x) \boldsymbol{u}(x) \cdot \boldsymbol{v}(x) d x \quad(\boldsymbol{u}, \boldsymbol{v} \in \mathbb{H})
$$


which defines an inner product in $\mathbb{H}$ making it a Hilbert space. The associated norm is denoted in the sequel by $|\cdot|_{\varepsilon}$ and is obviously equivalent to the natural norm of $\mathbb{H}$ denoted by $|\cdot|$.

Given $u_{0} \in \mathbb{H}$, we are interested in the asymptotic behavior of the sequence of velocity field $\left(\boldsymbol{u}_{\varepsilon}\right)_{\varepsilon>0}$ of the following generalized Ladyzhenskaya equation

$$
\begin{aligned}
& \rho^{\varepsilon} \frac{\partial \boldsymbol{u}_{\varepsilon}}{\partial t}-\operatorname{div}\left(a^{\varepsilon} \nabla \boldsymbol{u}_{\varepsilon}+b^{\varepsilon}\left|\nabla \boldsymbol{u}_{\varepsilon}\right|^{p-2} \nabla \boldsymbol{u}_{\varepsilon}\right)+\left(\boldsymbol{u}_{\varepsilon} \cdot \nabla\right) \boldsymbol{u}_{\varepsilon}+\nabla q_{\varepsilon}=\rho^{\varepsilon} f^{\varepsilon}\left(\cdot, \boldsymbol{u}_{\varepsilon}\right) \text { in } Q_{T} \\
& \operatorname{div} \boldsymbol{u}_{\varepsilon}=0 \operatorname{in} Q_{T} \\
& \boldsymbol{u}_{\varepsilon}=0 \text { on } \partial Q \times(0, T) \\
& \boldsymbol{u}_{\varepsilon}(x, 0)=\boldsymbol{u}^{0}(x) \text { in } Q .
\end{aligned}
$$

We introduce the functionals (for fixed $\varepsilon>0$ )

$$
a_{I}^{\varepsilon}(t ; \boldsymbol{u}, \boldsymbol{v})=\int_{Q}\left(a^{\varepsilon} \nabla \boldsymbol{u}\right) \cdot \nabla \boldsymbol{v} d x+\int_{Q} b^{\varepsilon}|\nabla \boldsymbol{u}|^{p-2} \nabla \boldsymbol{u} \cdot \nabla \boldsymbol{v} d x
$$

and

$$
b_{I}(\boldsymbol{u}, \boldsymbol{v}, \boldsymbol{w})=\sum_{i, j=1}^{N} \int_{Q} u_{i} \frac{\partial v_{j}}{\partial x_{i}} w_{j} d x
$$

defined for $\boldsymbol{u}, \boldsymbol{v}, \boldsymbol{w} \in W_{0}^{1, p}(Q)^{N}$. Then the following estimates hold:

$$
\begin{gathered}
\left|a_{I}^{\varepsilon}(t ; \boldsymbol{u}, \boldsymbol{v})\right| \leq\|a\|_{\infty}\|\nabla \boldsymbol{u}\|_{L^{2}(Q)}\|\nabla \boldsymbol{v}\|_{L^{2}(Q)}+\nu_{2}\|\nabla \boldsymbol{u}\|_{L^{p}(Q)}^{p-1}\|\nabla \boldsymbol{v}\|_{L^{p}(Q)}, \\
a_{I}^{\varepsilon}(t ; \boldsymbol{v}, \boldsymbol{v}) \geq \nu_{0}\|\nabla \boldsymbol{v}\|_{L^{2}(Q)}^{2}+\nu_{1}\|\nabla \boldsymbol{v}\|_{L^{p}(Q)}^{p}
\end{gathered}
$$

for all $\boldsymbol{u}, \boldsymbol{v} \in W_{0}^{1, p}(Q)^{N}$. Since $p \geq 1+\frac{2 N}{N+2}$ (hence $p \geq 2$ ) we have $L^{p}(Q) \hookrightarrow L^{2}(Q)$ so that, by the estimate (2.2) we infer the existence of an operator $\mathcal{A}^{\varepsilon}(t): \mathbb{V} \rightarrow \mathbb{V}^{\prime}$ such that

$$
a_{I}^{\varepsilon}(t ; \boldsymbol{u}, \boldsymbol{v})=\left\langle\mathcal{A}^{\varepsilon}(t) \boldsymbol{u}, \boldsymbol{v}\right\rangle \text { for all } \boldsymbol{u}, \boldsymbol{v} \in \mathbb{V} .
$$

Hence the existence of a bounded operator $\mathcal{A}^{\varepsilon}: L^{p}(0, T ; \mathbb{V}) \rightarrow L^{p^{\prime}}\left(0, T ; \mathbb{V}^{\prime}\right)$ such that

$$
\left\langle\mathcal{A}^{\varepsilon} \boldsymbol{u}, \boldsymbol{v}\right\rangle=\int_{0}^{T}\left\langle\mathcal{A}^{\varepsilon}(t) \boldsymbol{u}(t), \boldsymbol{v}(t)\right\rangle d t \text { for all } \boldsymbol{u}, \boldsymbol{v} \in L^{p}(0, T ; \mathbb{V}) .
$$

Now, dealing with the trilinear functional $b_{I}$, we have that [22, 38.

$$
\begin{aligned}
& b_{I}(\boldsymbol{u}, \boldsymbol{v}, \boldsymbol{v})=0 \text { for all } \boldsymbol{u} \in \mathbb{V} \text { and } \boldsymbol{v} \in W_{0}^{1, p}(Q)^{N} \\
& b_{I}(\boldsymbol{u}, \boldsymbol{u}, \boldsymbol{v})=-b_{I}(\boldsymbol{u}, \boldsymbol{v}, \boldsymbol{u}) \text { for all } \boldsymbol{u} \in \mathbb{V} \text { and } \boldsymbol{v} \in W_{0}^{1, p}(Q)^{N} .
\end{aligned}
$$

The following Sobolev embedding holds true [1]: $W^{1, p}(Q) \hookrightarrow L^{r}(Q)$ for $\frac{1}{r}=\frac{1}{p}-\frac{1}{N}$ if $\frac{1}{p}-\frac{1}{N}>0$, and for any $r>1$ if $\frac{1}{p}-\frac{1}{N} \leq 0$. In view of the choice of $p$ and $N$, we may always find $r>1$ such that $\frac{2}{r}+\frac{1}{p}=1$, and hence, using Hölder's inequality, we get

$$
\left|b_{I}(\boldsymbol{u}, \boldsymbol{u}, \boldsymbol{v})\right|=\left|b_{I}(\boldsymbol{u}, \boldsymbol{v}, \boldsymbol{u})\right| \leq c\|\boldsymbol{u}\|_{L^{r}(Q)}^{2}\|\nabla \boldsymbol{v}\|_{L^{p}(Q)}(\boldsymbol{u}, \boldsymbol{v} \in \mathbb{V}) .
$$

We infer from the above inequality the existence of an element $B(\boldsymbol{u}) \in \mathbb{V}^{\prime}$ such that

$$
\langle B(\boldsymbol{u}), \boldsymbol{v}\rangle=b_{I}(\boldsymbol{u}, \boldsymbol{u}, \boldsymbol{v}) \text { for all } \boldsymbol{u}, \boldsymbol{v} \in \mathbb{V} .
$$


This defines a bounded operator $B: \mathbb{V} \rightarrow \mathbb{V}^{\prime}$ verifying the further property that, if $\boldsymbol{u} \in L^{p}(0, T ; \mathbb{V})$ then $B(\boldsymbol{u}) \in L^{p^{\prime}}\left(0, T ; \mathbb{V}^{\prime}\right)$. Indeed taking $\boldsymbol{u} \in L^{p}(0, T ; \mathbb{V})$ we have by the Hölder's inequality

$$
\|B(\boldsymbol{u})\|_{L^{p^{\prime}\left(0, T ; \mathbb{V}^{\prime}\right)}} \leq\left(\int_{0}^{T}\|\boldsymbol{u}(t)\|_{L^{r}(Q)}^{2 p^{\prime}} d t\right)^{1 / p^{\prime}} .
$$

But since $W^{1, p}(Q) \hookrightarrow L^{r}(Q)$, there is a positive constant $c_{0}$ independent of $\boldsymbol{u}$ such that

$$
\|B(\boldsymbol{u})\|_{L^{p^{\prime}\left(0, T ; \mathbb{V}^{\prime}\right)}} \leq c_{0}\left(\int_{0}^{T}\|\boldsymbol{u}(t)\|^{2 p^{\prime}} d t\right)^{1 / p^{\prime}}
$$

where we recall that $\|\cdot\|$ stands for the norm in $\mathbb{V}$. We distinguish two situations: the case when $p \geq 3$ and the case when $1+\frac{2 N}{N+2} \leq p<3$. If $p \geq 3$, then $2 p^{\prime} \leq p$, hence the Hölder's inequality again gives (noting that $\frac{p}{2 p^{\prime}} \geq 1$ )

$$
\left(\int_{0}^{T}\|\boldsymbol{u}(t)\|^{2 p^{\prime}} d t\right)^{1 / p^{\prime}} \leq c_{1}\left(\int_{0}^{T}\|\boldsymbol{u}(t)\|^{p} d t\right)^{2 / p},
$$

so that

$$
\|B(\boldsymbol{u})\|_{L^{p^{\prime}\left(0, T ; \mathbb{V}^{\prime}\right)}} \leq c_{2}\|\boldsymbol{u}\|_{L^{p}(0, T ; \mathbb{V})}^{2} .
$$

Now, if $1+\frac{2 N}{N+2} \leq p<3$, then it is easy to get an inequality similar to (2.5) for $N=2$. So we shall only deal with the case $N=3$. With this in mind, returning to the inequality (2.4) which is true for $r=2 p^{\prime}$, we have

$$
\|B(\boldsymbol{u})\|_{L^{p^{\prime}\left(0, T ; \mathbb{V}^{\prime}\right)}} \leq c_{2}\left(\int_{0}^{T}\|\boldsymbol{u}(t)\|_{L^{2 p^{\prime}}(Q)}^{2 p^{\prime}} d t\right)^{2 / p} .
$$

But 20] (see also [18, Lemma 4.1])

$$
\|u\|_{L^{2 p^{\prime}}(Q)} \leq C\|u\|_{W^{1, p}(Q)}^{\alpha}\|u\|_{L^{2}(Q)}^{1-\alpha} \text { for any } u \in W^{1, p}(Q)
$$

where $\alpha=3 /(5 p-6)$. But if $\boldsymbol{u} \in L^{p}(0, T ; \mathbb{V}) \cap L^{\infty}(0, T ; \mathbb{H})$, the function $t \mapsto$ $\|\boldsymbol{u}(t)\|^{2 \alpha}|\boldsymbol{u}(t)|^{2-2 \alpha}$ lies in $L^{r_{0}}(0, T)$ for $r_{0} \geq 1$ satisfying $\frac{1}{r_{0}}=\frac{2 \alpha}{p}+\frac{1}{\infty}$, and more generally, in any $L^{r}(0, T)$ for $1<r \leq \frac{p}{2 \alpha}$. Since $p^{\prime} \leq \frac{p}{2 \alpha}$ (for $3>p \geq \frac{11}{5}$ ) we have that it belongs to $L^{p^{\prime}}(0, T)$. Using Hölder's inequality in (2.6) (with exponent $r=\frac{p}{2 \alpha p^{\prime}}$ ) we have

$$
\|B(\boldsymbol{u})\|_{L^{p^{\prime}}\left(0, T ; \mathbb{V}^{\prime}\right)}^{p^{\prime}} \leq C\left(\int_{0}^{T}\|\boldsymbol{u}(t)\|^{p} d t\right)^{\frac{2 \alpha p^{\prime}}{p}}\left(\int_{0}^{T}|\boldsymbol{u}(t)|^{(2-2 \alpha) p^{\prime} r^{\prime}} d t\right)^{\frac{1}{r^{\prime}}}
$$

(where $r^{\prime}=r /(r-1)$ ), or equivalently,

$$
\|B(\boldsymbol{u})\|_{L^{p^{\prime}\left(0, T ; \mathbb{V}^{\prime}\right)}} \leq C\|\boldsymbol{u}\|_{L^{p}(0, T ; \mathbb{V})}^{2 \alpha}\|\boldsymbol{u}\|_{L^{\infty}(0, T ; \mathbb{H})}^{2-2 \alpha}
$$

for any $\boldsymbol{u} \in L^{p}(0, T ; \mathbb{V}) \cap L^{\infty}(0, T ; \mathbb{H})$.

We have proven the following inequalities:

$$
\left\{\begin{array}{l}
\text { If } p \geq 3,\|B(\boldsymbol{u})\|_{L^{p^{\prime}}\left(0, T ; \mathbb{V}^{\prime}\right)} \leq c_{2}\|\boldsymbol{u}\|_{L^{p}(0, T ; \mathbb{V})}^{2} \text { for all } \boldsymbol{u} \in L^{p}(0, T ; \mathbb{V}) \\
\text { If } 1+\frac{2 N}{N+2} \leq p<3,\|B(\boldsymbol{u})\|_{L^{p^{\prime}}\left(0, T ; \mathbb{V}^{\prime}\right)} \leq C\|\boldsymbol{u}\|_{L^{p}(0, T ; \mathbb{V})}^{2 \alpha}\|\boldsymbol{u}\|_{L^{\infty}(0, T ; \mathbb{H})}^{2-2 \alpha} \\
\text { for all } \boldsymbol{u} \in L^{p}(0, T ; \mathbb{V}) \cap L^{\infty}(0, T ; \mathbb{H}) .
\end{array}\right.
$$


The above inequalities will be very useful in the sequel. For the sake of completeness, we choose $\boldsymbol{u}^{0} \in \mathbb{H}$. We are therefore concerned with the existence of a solution of (2.1). The first result of the work is the following

Theorem 1. Let $1+\frac{2 N}{N+2} \leq p<\infty$. Suppose $\boldsymbol{u}^{0} \in \mathbb{H}$. Under assumptions (A1)-(A4), there exists (for each fixed $\varepsilon>0)$ a couple $\left(\boldsymbol{u}_{\varepsilon}, q_{\varepsilon}\right) \in L^{p}(0, T ; \mathbb{V}) \cap$ $L^{\infty}(0, T ; \mathbb{H}) \times W^{-1, \infty}\left(0, T ; L^{p^{\prime}}(Q)\right)$ solution to (2.1). The function $\boldsymbol{u}_{\varepsilon}$ also belongs to $\mathcal{C}([0, T] ; \mathbb{H})$ and $q_{\varepsilon}$ is unique up to a constant function of $x: \int_{Q} q_{\varepsilon} d x=0$.

Proof. Multiplying Eq. (2.1) by $\boldsymbol{v} \in \mathbb{V}$ and integrating over $(0, t) \times Q$ we get

$$
\left\{\begin{array}{l}
\left(\boldsymbol{u}_{\varepsilon}(t), \boldsymbol{v}\right)_{\varepsilon}+\int_{0}^{t}\left\langle\mathcal{A}^{\varepsilon}(s) \boldsymbol{u}_{\varepsilon}(s)+B\left(\boldsymbol{u}_{\varepsilon}(s)\right), \boldsymbol{v}\right\rangle d s=\left(\boldsymbol{u}^{0}, \boldsymbol{v}\right)_{\varepsilon} \\
+\int_{0}^{t}\left(f\left(\frac{s}{\varepsilon^{2}}, \boldsymbol{u}_{\varepsilon}(s)\right), \boldsymbol{v}\right)_{\varepsilon} d s \text { for all } \boldsymbol{v} \in \mathbb{V} \text { and a.e. } 0<t<T,
\end{array}\right.
$$

which, in view of the properties of the operators $\mathcal{A}^{\varepsilon}$ and $B$ (see especially (2.8)), amounts to find a function $\boldsymbol{u}_{\varepsilon} \in L^{p}(0, T ; \mathbb{V})$ such that

$$
\frac{d \boldsymbol{u}_{\varepsilon}}{d t}+\mathcal{A}^{\varepsilon} \boldsymbol{u}_{\varepsilon}+B\left(\boldsymbol{u}_{\varepsilon}\right)=f^{\varepsilon}\left(\cdot, \boldsymbol{u}_{\varepsilon}\right) \text { in } L^{p^{\prime}}\left(0, T ; \mathbb{V}^{\prime}\right), \boldsymbol{u}_{\varepsilon}(0)=\boldsymbol{u}^{0} \text { in } \mathbb{H} .
$$

Conversely, a solution of (2.9) will satisfy (2.1) for a suitable choice of $q_{\varepsilon}$ which shall be specified later. Therefore, in view of the properties of the operators $\mathcal{A}^{\varepsilon}$ and $B$ (see once again (2.8)), we can argue as in [8, Theorem 2.1] (see also [9]) to get the existence of a solution to (2.1) in the space $L^{p}(0, T ; \mathbb{V}) \cap L^{\infty}(0, T ; \mathbb{H})$. It is to be noted that the solution $\boldsymbol{u}_{\varepsilon}$ satisfies $\frac{\partial \boldsymbol{u}_{\varepsilon}}{\partial t} \in L^{p^{\prime}}\left(0, T ; \mathbb{V}^{\prime}\right)$, so that, by a well-known result, $\boldsymbol{u}_{\varepsilon} \in \mathcal{C}([0, T] ; \mathbb{H})$.

For the existence of the pressure, we have $\rho^{\varepsilon} f^{\varepsilon}\left(\cdot, \boldsymbol{u}_{\varepsilon}\right) \in L^{2}\left(Q_{T}\right)^{N}$, so that the necessary condition of [35, Section 4] for the existence of the pressure is satisfied. Now, coming back to (2.1) and denoting there

$$
\boldsymbol{w}_{\varepsilon}=\rho^{\varepsilon} f^{\varepsilon}\left(\cdot, \boldsymbol{u}_{\varepsilon}\right)-\rho^{\varepsilon} \frac{\partial \boldsymbol{u}_{\varepsilon}}{\partial t}+\operatorname{div}\left(a^{\varepsilon} \nabla \boldsymbol{u}_{\varepsilon}+b^{\varepsilon}\left|\nabla \boldsymbol{u}_{\varepsilon}\right|^{p-2} \nabla \boldsymbol{u}_{\varepsilon}\right)-\left(\boldsymbol{u}_{\varepsilon} \cdot \nabla\right) \boldsymbol{u}_{\varepsilon}
$$

we have $\left\langle\boldsymbol{w}_{\varepsilon}, \boldsymbol{v}\right\rangle=0$ for all $\boldsymbol{v} \in \mathcal{V}$ (that is for all $\boldsymbol{v} \in \mathcal{C}_{0}^{\infty}(Q)^{N}$ with $\operatorname{div} \boldsymbol{v}=0$ ) where $\langle\cdot, \cdot\rangle$ is the duality pairing between $\mathcal{D}^{\prime}(Q)^{N}$ and $\mathcal{D}(Q)^{N}$. Next, arguing as in the proof of [35. Proposition 5] we are led to $\boldsymbol{w}_{\varepsilon} \in W^{-1, \infty}\left(0, T ; W^{-1, p^{\prime}}(Q)^{N}\right)$, so that there exists a unique $q_{\varepsilon} \in W^{-1, \infty}\left(0, T ; L^{p^{\prime}}(Q)\right)$ such that

$$
\nabla q_{\varepsilon}=\boldsymbol{w}_{\varepsilon}, \int_{Q} q_{\varepsilon} d x=0
$$

This completes the proof.

2.2. A priori estimates and compactness. The following result holds.

Lemma 1. Let $1+\frac{2 N}{N+2} \leq p<\infty$. Under assumptions (A1)-(A4) we have the following estimates:

$$
\begin{aligned}
& \sup _{0 \leq t \leq T}\left|\boldsymbol{u}_{\varepsilon}(t)\right|^{2} \leq C, \\
& \int_{0}^{T}\left\|\boldsymbol{u}_{\varepsilon}(t)\right\|^{p} d t \leq C
\end{aligned}
$$

where $C$ is a positive constant which does not depends on $\varepsilon$. 
Proof. The variational formulation of (2.1) gives, for any $\boldsymbol{v} \in \mathbb{V}$,

$$
\frac{d}{d t}\left(\boldsymbol{u}_{\varepsilon}(t), \boldsymbol{v}\right)_{\varepsilon}+a_{I}^{\varepsilon}\left(t ; \boldsymbol{u}_{\varepsilon}(t), \boldsymbol{v}\right)+b_{I}\left(\boldsymbol{u}_{\varepsilon}(t), \boldsymbol{u}_{\varepsilon}(t), \boldsymbol{v}\right)=\left(f\left(\frac{t}{\varepsilon^{2}}, \boldsymbol{u}_{\varepsilon}(t)\right), \boldsymbol{v}\right)_{\varepsilon} .
$$

In all what follows, $C$ is a generic constant that may change from line to line.

Taking the particular $\boldsymbol{v}=\boldsymbol{u}_{\varepsilon}(t)$ in (2.12) and using the relation $b_{I}\left(\boldsymbol{u}_{\varepsilon}(t), \boldsymbol{u}_{\varepsilon}(t), \boldsymbol{u}_{\varepsilon}(t)\right)=$ 0 , we get (after integrating over $[0, t]$ ) for all $t \in[0, T]$,

$$
\begin{aligned}
& \left|\boldsymbol{u}_{\varepsilon}(t)\right|_{\varepsilon}^{2}+2 \nu_{0} \int_{0}^{t}\left\|\boldsymbol{u}_{\varepsilon}(s)\right\|_{H_{0}^{1}(Q)^{N}}^{2} d s+2 \nu_{1} \int_{0}^{t}\left\|\boldsymbol{u}_{\varepsilon}(s)\right\|^{p} d s \\
\leq & \left|\boldsymbol{u}^{0}\right|_{\varepsilon}^{2}+2 \int_{0}^{t}\left|f\left(\frac{s}{\varepsilon^{2}}, \boldsymbol{u}_{\varepsilon}(s)\right)\right|_{\varepsilon}\left|\boldsymbol{u}_{\varepsilon}(s)\right|_{\varepsilon} d s .
\end{aligned}
$$

From assumption (A4) we have that

$$
\left|f\left(\frac{s}{\varepsilon^{2}}, \boldsymbol{u}_{\varepsilon}(s)\right)\right|_{\varepsilon} \leq C\left(1+\left|\boldsymbol{u}_{\varepsilon}(s)\right|_{\varepsilon}\right),
$$

hence

$$
\begin{aligned}
2 \int_{0}^{t}\left|f\left(\frac{s}{\varepsilon^{2}}, \boldsymbol{u}_{\varepsilon}(s)\right)\right|_{\varepsilon}\left|\boldsymbol{u}_{\varepsilon}(s)\right|_{\varepsilon} d s & \leq C \int_{0}^{t}\left(1+\left|\boldsymbol{u}_{\varepsilon}(s)\right|_{\varepsilon}\right)\left|\boldsymbol{u}_{\varepsilon}(s)\right|_{\varepsilon} d s \\
& \leq C+C \int_{0}^{t}\left|\boldsymbol{u}_{\varepsilon}(s)\right|_{\varepsilon}^{2} d s
\end{aligned}
$$

We therefore get

$$
\begin{aligned}
& \left|\boldsymbol{u}_{\varepsilon}(t)\right|_{\varepsilon}^{2}+2 \nu_{0} \int_{0}^{t}\left\|\boldsymbol{u}_{\varepsilon}(s)\right\|_{H_{0}^{1}(Q)^{N}}^{2} d s+2 \nu_{1} \int_{0}^{t}\left\|\boldsymbol{u}_{\varepsilon}(s)\right\|^{p} d s \\
& \quad \leq C+C \int_{0}^{t}\left|\boldsymbol{u}_{\varepsilon}(s)\right|_{\varepsilon}^{2} d s
\end{aligned}
$$

since $\sup _{\varepsilon>0}\left|\boldsymbol{u}^{0}\right|_{\varepsilon}^{2}<\infty$. We readily deduce from (2.13)

$$
\left|\boldsymbol{u}_{\varepsilon}(t)\right|_{\varepsilon}^{2} \leq C+C \int_{0}^{t}\left|\boldsymbol{u}_{\varepsilon}(s)\right|_{\varepsilon}^{2} d s .
$$

By the application of Gronwall's inequality we find that

$$
\left|\boldsymbol{u}_{\varepsilon}(t)\right|_{\varepsilon}^{2} \leq C \text { for } 0 \leq t \leq T, \varepsilon>0
$$

where $C$ is a positive constant independent of $\varepsilon$ and $t$. But $\left|\boldsymbol{u}_{\varepsilon}(t)\right|_{\varepsilon}^{2} \geq \Lambda^{-1}\left|\boldsymbol{u}_{\varepsilon}(t)\right|^{2}$, so that (2.10) comes from (2.14). We also infer from (2.13) that (2.11) holds true. Still from (2.13) it emerges that

$$
\sup _{\varepsilon>0} \int_{0}^{T}\left\|\boldsymbol{u}_{\varepsilon}(t)\right\|_{H_{0}^{1}(Q)^{N}}^{2} d t \leq C .
$$

The next result will be of great interest in the homogenization process.

Proposition 1. The sequence $\left(\boldsymbol{u}_{\varepsilon}\right)_{\varepsilon>0}$ is relatively compact in the space $L^{2}\left(Q_{T}\right)^{N}$.

Proof. Let

$$
\mathbb{W}=\left\{\boldsymbol{u} \in L^{p}(0, T ; \mathbb{V}): \frac{\partial \boldsymbol{u}}{\partial t} \in L^{p^{\prime}}\left(0, T ; \mathbb{V}^{\prime}+L^{2}(Q)^{N}\right)\right\} .
$$

Since $\mathbb{V} \hookrightarrow \mathbb{H}$ is compact and $\mathbb{H} \hookrightarrow L^{2}(Q)^{N}$, we have that $\mathbb{V} \hookrightarrow L^{2}(Q)^{N}$ is compact. We also have $L^{2}(Q)^{N} \hookrightarrow \mathbb{V}^{\prime}+L^{2}(Q)^{N}$, hence the compactness of $\mathbb{W}$ in $L^{2}\left(0, T ; L^{2}(Q)^{N}\right)=L^{2}\left(Q_{T}\right)^{N}$ by a well-known result; see e.g. 222, p. 58, Theorem 
5.1]. With this in mind we need to check that the sequence $\left(\boldsymbol{u}_{\varepsilon}\right)_{\varepsilon>0}$ is bounded in $\mathbb{W}$. But since $\mathbb{V}^{\prime} \hookrightarrow \mathbb{V}^{\prime}+L^{2}(Q)^{N}$ we just need to verify (after (2.11)) that $\left(\partial \boldsymbol{u}_{\varepsilon} / \partial t\right)_{\varepsilon>0}$ is bounded in $L^{p^{\prime}}\left(0, T ; \mathbb{V}^{\prime}\right)$. But this comes from the combination of (2.10)-(2.11) with (2.8) and (2.15).

We can now deal with the estimation of the pressure. Before we can do that, let us recall the definition and properties of the Bogovskii operator. Let

$$
L_{0}^{p}(Q)=\left\{v \in L^{p}(Q): \int_{Q} v d x=0\right\}
$$

for $1<p<\infty$. We have the following result.

Lemma 2 ([27, Lemma 3.17, p.169]). Let $1<p<\infty$. There exists a linear operator $\mathcal{B}: L_{0}^{p}(Q) \rightarrow W_{0}^{1, p}(Q)^{N}$ with the following properties:

(i) $\operatorname{div} \mathcal{B}(f)=f$ a.e. in $Q$ for any $f \in L_{0}^{p}(Q)$

(ii) $\|\mathcal{B}(f)\|_{W_{0}^{1, p}(Q)^{N}} \leq c(p, Q)\|f\|_{L^{p}(Q)}$

(iii) If $f=\operatorname{div} g$ with $g \in L^{r}(Q)^{N}$ and $g \cdot n=0$ on $\partial Q$ for some $1<r<\infty$ where $n$ is an outward unit vector normal to $\partial Q$, then

$$
\|\mathcal{B}(f)\|_{L^{r}(Q)^{N}} \leq c(r, Q)\|g\|_{L^{r}(Q)^{N}} .
$$

(iv) If $f \in \mathcal{C}_{0}^{\infty}(Q) \cap L_{0}^{p}(Q)$ then $\mathcal{B}(f) \in \mathcal{C}_{0}^{\infty}(Q)^{N}$.

With this in mind, the following result holds.

Lemma 3. Let $1+\frac{2 N}{N+2} \leq p<\infty$ and $p^{\prime}$ its conjugate. We have $q_{\varepsilon} \in L^{p^{\prime}}\left(0, T ; L_{0}^{p^{\prime}}(Q)\right)$ with

$$
\sup _{\varepsilon>0}\left\|q_{\varepsilon}\right\|_{L^{p^{\prime}}\left(Q_{T}\right)} \leq C
$$

for some positive constant $C$ independent of $\varepsilon$.

Proof. First we know that $q_{\varepsilon} \in \mathcal{D}^{\prime}\left(Q_{T}\right)$. Let $g \in \mathcal{C}_{0}^{\infty}\left(Q_{T}\right) \cap L^{p}\left(0, T ; L_{0}^{p}(Q)\right.$ ) (recall that the dual of $L_{0}^{p^{\prime}}(Q)$ is $\left.L_{0}^{p}(Q)\right)$. In view of [part (iv) of] Lemma 2 let $\boldsymbol{v} \in$ $\mathcal{C}_{0}^{\infty}\left(Q_{T}\right)^{N}$ be such that $\operatorname{div} \boldsymbol{v}=g$. We have

$$
\left\langle\nabla q_{\varepsilon}, \boldsymbol{v}\right\rangle=-\left\langle q_{\varepsilon}, \operatorname{div} \boldsymbol{v}\right\rangle=-\left\langle q_{\varepsilon}, g\right\rangle,
$$

that is (because of (2.1))

$$
\left\langle q_{\varepsilon}, g\right\rangle=-\left\langle\nabla q_{\varepsilon}, \boldsymbol{v}\right\rangle=\left\langle-\boldsymbol{w}_{\varepsilon}, \boldsymbol{v}\right\rangle
$$

where

$$
\boldsymbol{w}_{\varepsilon}=\rho^{\varepsilon} f^{\varepsilon}\left(\cdot, \boldsymbol{u}_{\varepsilon}\right)-\rho^{\varepsilon} \frac{\partial \boldsymbol{u}_{\varepsilon}}{\partial t}+\operatorname{div}\left(a^{\varepsilon} \nabla \boldsymbol{u}_{\varepsilon}+b^{\varepsilon}\left|\nabla \boldsymbol{u}_{\varepsilon}\right|^{p-2} \nabla \boldsymbol{u}_{\varepsilon}\right)-\left(\boldsymbol{u}_{\varepsilon} \cdot \nabla\right) \boldsymbol{u}_{\varepsilon} .
$$

But we infer from Lemma 1 that

$$
\begin{aligned}
\left|\left\langle q_{\varepsilon}, g\right\rangle\right| & \leq C\left(\|v\|_{L^{p}\left(0, T ; W_{0}^{1, p}(Q)^{N}\right)}+\|v\|_{L^{p}\left(Q_{T}\right)^{N}}\right) \\
& \leq C\|\boldsymbol{v}\|_{L^{p}\left(0, T ; W_{0}^{1, p}(Q)^{N}\right)} \\
& \leq C\|g\|_{L^{p}\left(Q_{T}\right)},
\end{aligned}
$$

the last inequality above being due to [part (ii) of] Lemma 2, We therefore deduce from the above inequality that $q_{\varepsilon} \in L^{p^{\prime}}\left(Q_{T}\right)$, that is $q_{\varepsilon} \in L^{p^{\prime}}\left(0, T ; L_{0}^{p^{\prime}}(Q)\right)$ with

$$
\left\|q_{\varepsilon}\right\|_{L^{p^{\prime}}\left(Q_{T}\right)} \leq C
$$

for a constant $C>0$ independent of $\varepsilon$. 


\section{The $\Sigma$-CONVERGENCE METHOD}

We begin this section by collecting some useful tools about almost periodicity.

3.1. Almost periodic functions. The concept of almost periodic functions is well known in the literature. Following [29, Section 2], we present in this section some basic facts about it, which will be used throughout the paper. For a general presentation and an efficient treatment of this concept, we refer to [5], [3] and [21].

Let $\mathcal{B}\left(\mathbb{R}^{N}\right)$ denote the Banach algebra of bounded continuous (complex-valued) functions on $\mathbb{R}^{N}$ endowed with the sup norm topology.

A mapping $u: \mathbb{R}^{N} \rightarrow \mathbb{C}$ is called an almost periodic function, or a Bohr almost periodic function, if $u \in \mathcal{B}\left(\mathbb{R}^{N}\right)$ and further the set of all its translates $\{u(\cdot+a)$ : $\left.a \in \mathbb{R}^{N}\right\}$ has a compact closure in $\mathcal{B}\left(\mathbb{R}^{N}\right)$. We denote by $A P\left(\mathbb{R}^{N}\right)$ the set of all continuous almost periodic functions on $\mathbb{R}^{N} . A P\left(\mathbb{R}^{N}\right)$ is a commutative $\mathcal{C}^{*}$-algebra with identity. Next, let us denote by $\operatorname{Trig}\left(\mathbb{R}^{N}\right)$ the algebra of all trigonometric polynomials, i.e. all finite sums of the form

$$
u(y)=\sum a_{k} \exp \left(i \xi_{k} \cdot y\right), \xi_{k} \in \mathbb{R}^{N}, a_{k} \in \mathbb{C} .
$$

Then $\operatorname{Trig}\left(\mathbb{R}^{N}\right) \subset A P\left(\mathbb{R}^{N}\right)$ and further $A P\left(\mathbb{R}^{N}\right)$ coincides with the closure of $\operatorname{Trig}\left(\mathbb{R}^{N}\right)$ in $\mathcal{B}\left(\mathbb{R}^{N}\right)$. From the above definition, one easily sees that every element of $A P\left(\mathbb{R}^{N}\right)$ is uniformly continuous. Moreover it is classically known that $A P\left(\mathbb{R}^{N}\right)$ enjoys the following properties:

$(\mathrm{P})_{1} u(\cdot+a) \in A P\left(\mathbb{R}^{N}\right)$ whenever $u \in A P\left(\mathbb{R}^{N}\right)$ and for every $a \in \mathbb{R}^{N}$;

$(\mathrm{P})_{2}$ For each $u \in A P\left(\mathbb{R}^{N}\right)$ the closed convex hull of $\{u(\cdot+a)\}_{a \in \mathbb{R}^{N}}$ in $\mathcal{B}\left(\mathbb{R}^{N}\right)$ contains a unique complex constant $\mathfrak{M}(u)$ called the mean value of $u$, and which satisfies the property that the sequence $\left(u^{\varepsilon}\right)_{\varepsilon>0}$ (where $u^{\varepsilon}(x)=u(x / \varepsilon)$, $\left.x \in \mathbb{R}^{N}\right)$ weakly $*$-converges in $L^{\infty}\left(\mathbb{R}^{N}\right)$ to $\mathfrak{M}(u)$ as $\varepsilon \rightarrow 0$.

It follows from the above properties that $A P\left(\mathbb{R}^{N}\right)$ is an algebra with mean value on $\mathbb{R}^{N}$ [19]. Its spectrum is the Bohr compactification of $\mathbb{R}^{N}$, sometimes denoted by $b \mathbb{R}^{N}$ in the literature and, in order to simplify the notation, we denote it here just by $\mathcal{K}$. The set $\mathcal{K}$ is a compact topological Abelian group. The Haar measure on $\mathcal{K}$ is denoted by $\beta$. In view of the Gelfand representation theory of $\mathcal{C}^{*}$-algebras we have the next result.

Theorem 2. There exists an isometric *-isomorphism $\mathcal{G}$ of $A P\left(\mathbb{R}^{N}\right)$ onto $\mathcal{C}(\mathcal{K})$ such that every element of $A P\left(\mathbb{R}^{N}\right)$ is viewed as a restriction to $\mathbb{R}^{N}$ of a unique element in $\mathcal{C}(\mathcal{K})$. Moreover the mean value $\mathfrak{M}$ defined on $A P\left(\mathbb{R}^{N}\right)$ has an integral representation in terms of the Haar measure $\beta$ as follows:

$$
\mathfrak{M}(u)=\int_{\mathcal{K}} \mathcal{G}(u) d \beta \text { for all } u \in A P\left(\mathbb{R}^{N}\right) .
$$

The isometric $*$-isomorphism $\mathcal{G}$ of the above theorem is referred to as the Gelfand transformation. The image $\mathcal{G}(u)$ of $u$ will very often be denoted by $\widehat{u}$.

We introduce the space $A P^{\infty}\left(\mathbb{R}^{N}\right)=\left\{u \in A P\left(\mathbb{R}^{N}\right): D_{y}^{\alpha} u \in A P\left(\mathbb{R}^{N}\right)\right.$ for every $\left.\alpha=\left(\alpha_{1}, \ldots, \alpha_{N}\right) \in \mathbb{N}^{N}\right\}$ where $D_{y}^{\alpha}=\frac{\partial^{|\alpha|}}{\partial y_{1}^{\alpha_{1}} \ldots \partial y_{N}^{\alpha_{N}}}$. For $m \in \mathbb{N}$ (the non negative integers) and for $u \in A P^{\infty}\left(\mathbb{R}^{N}\right)$ we set $\||u|\|_{m}=\sup _{|\alpha| \leq m} \sup _{y \in \mathbb{R}^{N}}\left|D_{y}^{\alpha} u\right|$ (which defines a norm on $\left.A P^{\infty}\left(\mathbb{R}^{N}\right)\right)$. Then $A P^{\infty}\left(\mathbb{R}^{N}\right)$ is a Fréchet space with respect to the natural topology of projective limit, defined by the increasing family of norms $\||\cdot|\|_{m}(m \in \mathbb{N})$. 
Next, let $B_{A P}^{p}\left(\mathbb{R}^{N}\right)(1 \leq p<\infty)$ denote the space of Besicovitch almost periodic functions on $\mathbb{R}^{N}$, that is the closure of $A P\left(\mathbb{R}^{N}\right)$ with respect to the Besicovitch seminorm

$$
\|u\|_{p}=\left(\limsup _{r \rightarrow+\infty} \frac{1}{\left|B_{r}\right|} \int_{B_{r}}|u(y)|^{p} d y\right)^{1 / p}
$$

where $B_{r}$ is the open ball of $\mathbb{R}^{N}$ of radius $r$ centered at the origin. It is known that $B_{A P}^{p}\left(\mathbb{R}^{N}\right)$ is a complete seminormed vector space verifying $B_{A P}^{q}\left(\mathbb{R}^{N}\right) \subset B_{A P}^{p}\left(\mathbb{R}^{N}\right)$ for $1 \leq p \leq q<\infty$. Using this last property one may naturally define the space $B_{A P}^{\infty}\left(\mathbb{R}^{N}\right)$ as follows:

$$
B_{A P}^{\infty}\left(\mathbb{R}^{N}\right)=\left\{f \in \cap_{1 \leq p<\infty} B_{A P}^{p}\left(\mathbb{R}^{N}\right): \sup _{1 \leq p<\infty}\|f\|_{p}<\infty\right\} .
$$

We endow $B_{A P}^{\infty}\left(\mathbb{R}^{N}\right)$ with the seminorm $[f]_{\infty}=\sup _{1 \leq p<\infty}\|f\|_{p}$, which makes it a complete seminormed space. We recall that the spaces $B_{A P}^{p}\left(\mathbb{R}^{N}\right)(1 \leq p \leq \infty)$ are not Fréchet spaces since they are not separated. The following properties are worth noticing [24, 28]:

(1) The Gelfand transformation $\mathcal{G}: A P\left(\mathbb{R}^{N}\right) \rightarrow \mathcal{C}(\mathcal{K})$ extends by continuity to a unique continuous linear mapping, still denoted by $\mathcal{G}$, of $B_{A P}^{p}\left(\mathbb{R}^{N}\right)$ into $L^{p}(\mathcal{K})$, which in turn induces an isometric isomorphism $\mathcal{G}_{1}$, of $B_{A P}^{p}\left(\mathbb{R}^{N}\right) / \mathcal{N}=$ $\mathcal{B}_{A P}^{p}\left(\mathbb{R}^{N}\right)$ onto $L^{p}(\mathcal{K})$ (where $\mathcal{N}=\left\{u \in B_{A P}^{p}\left(\mathbb{R}^{N}\right): \mathcal{G}(u)=0\right\}$ ). Moreover if $u \in B_{A P}^{p}\left(\mathbb{R}^{N}\right) \cap L^{\infty}\left(\mathbb{R}^{N}\right)$ then $\mathcal{G}(u) \in L^{\infty}(\mathcal{K})$ and $\|\mathcal{G}(u)\|_{L^{\infty}(\mathcal{K})} \leq$ $\|u\|_{L^{\infty}\left(\mathbb{R}^{N}\right)}$.

(2) The mean value $\mathfrak{M}$, defined on $A P\left(\mathbb{R}^{N}\right)$, extends by continuity to a positive continuous linear form (still denoted by $\mathfrak{M})$ on $B_{A P}^{p}\left(\mathbb{R}^{N}\right)$ satisfying $\mathfrak{M}(u)=$ $\int_{\mathcal{K}} \mathcal{G}(u) d \beta$ and $\mathfrak{M}(u(\cdot+a))=\mathfrak{M}(u)$ for each $u \in B_{A P}^{p}\left(\mathbb{R}^{N}\right)$ and all $a \in \mathbb{R}^{N}$, where $u(\cdot+a)(y)=u(y+a)$ for almost all $y \in \mathbb{R}^{N}$. Moreover for $u \in$ $B_{A P}^{p}\left(\mathbb{R}^{N}\right)$ we have $\|u\|_{p}=\left[\mathfrak{M}\left(|u|^{p}\right)\right]^{1 / p}$.

Spaces of almost periodic functions with values in a Banach space are defined in a natural way, we refer to [4] for details. Keep the following notations in mind: $A P\left(\mathbb{R}^{N} ; \mathbb{C}\right)=A P\left(\mathbb{R}^{N}\right)$ and $B_{A P}^{p}\left(\mathbb{R}^{N} ; \mathbb{C}\right)=B_{A P}^{p}\left(\mathbb{R}^{N}\right)$.

We can also define the notion of almost periodic distributions. To do this, let $\mathcal{B}_{\infty}\left(\mathbb{R}^{N}\right)$ denote the space of all $\mathcal{C}^{\infty}$ functions in $\mathbb{R}^{N}$ that are bounded together with all their derivatives of any order. We equip $\mathcal{B}_{\infty}\left(\mathbb{R}^{N}\right)$ with the locally convex topology defined by the increasingly filtered separating family of norms $\||\cdot|\|_{m}(m \in$ $\mathbb{N})$. As usual, let $\mathcal{D}\left(\mathbb{R}^{N}\right)$ denote the subspace of $\mathcal{B}_{\infty}\left(\mathbb{R}^{N}\right)$ consisting of functions with compact support. We denote by $\mathcal{B}_{\infty}^{\prime}\left(\mathbb{R}^{N}\right)$ the topological dual of $\mathcal{B}_{\infty}\left(\mathbb{R}^{N}\right)$. We recall that $\mathcal{B}_{\infty}^{\prime}\left(\mathbb{R}^{N}\right)$ is not a subspace of $\mathcal{D}^{\prime}\left(\mathbb{R}^{N}\right)$ (the usual space of distributions on $\mathbb{R}^{N}$ ) since $\mathcal{D}\left(\mathbb{R}^{N}\right)$ is not dense in $\mathcal{B}_{\infty}\left(\mathbb{R}^{N}\right)$; see 33 . We endow $\mathcal{B}_{\infty}^{\prime}\left(\mathbb{R}^{N}\right)$ with the strong dual topology. For a distribution $T \in \mathcal{D}^{\prime}\left(\mathbb{R}^{N}\right)$ we define its translate $\tau_{a} T\left(a \in \mathbb{R}^{N}\right)$ as follows:

$$
\left\langle\tau_{a} T, \varphi\right\rangle=\langle T, \varphi(\cdot-a)\rangle \text { for any } \varphi \in \mathcal{D}\left(\mathbb{R}^{N}\right)
$$

With this in mind, we say that a distribution $T$ is an almost periodic distribution if $T \in \mathcal{B}_{\infty}^{\prime}\left(\mathbb{R}^{N}\right)$ and further the set of translates $\left\{\tau_{a} T: a \in \mathbb{R}^{N}\right\}$ is relatively compact in $\mathcal{B}_{\infty}^{\prime}\left(\mathbb{R}^{N}\right)$. We denote by $\mathcal{B}_{A P}^{\prime}\left(\mathbb{R}^{N}\right)$ the space of all almost periodic distributions on $\mathbb{R}^{N}$. $\mathcal{B}_{A P}^{\prime}\left(\mathbb{R}^{N}\right)$ is endowed with the relative topology on $\mathcal{B}_{\infty}^{\prime}\left(\mathbb{R}^{N}\right)$. The following result holds [33, p. 206, Section 9]. 
Proposition 2. The following assertions are equivalent.

(i) $T \in \mathcal{B}_{A P}^{\prime}\left(\mathbb{R}^{N}\right)$;

(ii) $T \in \mathcal{B}_{\infty}^{\prime}\left(\mathbb{R}^{N}\right)$ is a finite sum of derivatives of functions in $A P\left(\mathbb{R}^{N}\right)$;

(iii) $T \in \mathcal{B}_{\infty}^{\prime}\left(\mathbb{R}^{N}\right)$ and $T * \varphi \in A P^{\infty}\left(\mathbb{R}^{N}\right)$ for any $\varphi \in \mathcal{D}\left(\mathbb{R}^{N}\right)$.

It can be easily checked that $\varphi T \in \mathcal{B}_{A P}^{\prime}\left(\mathbb{R}^{N}\right)$ whenever $\varphi \in A P^{\infty}\left(\mathbb{R}^{N}\right)$ and $T \in \mathcal{B}_{A P}^{\prime}\left(\mathbb{R}^{N}\right)$. It follows from part (iii) of the above proposition that $A P^{\infty}\left(\mathbb{R}^{N}\right)$ is dense in $\mathcal{B}_{A P}^{\prime}\left(\mathbb{R}^{N}\right)$. Since $B_{A P}^{1}\left(\mathbb{R}^{N}\right)$ is the closure of $A P^{\infty}\left(\mathbb{R}^{N}\right)$ in the Besicovitch seminorm whereas $\mathcal{B}_{A P}^{\prime}\left(\mathbb{R}^{N}\right)$ is the closure of $A P^{\infty}\left(\mathbb{R}^{N}\right)$ in $\mathcal{B}_{\infty}^{\prime}\left(\mathbb{R}^{N}\right)$ it follows that $B_{A P}^{1}\left(\mathbb{R}^{N}\right)$ is continuously embedded in $\mathcal{B}_{A P}^{\prime}\left(\mathbb{R}^{N}\right)$. As a first consequence of this, we have the

Lemma 4. The mean value $\mathfrak{M}$ considered as defined on $A P^{\infty}\left(\mathbb{R}^{N}\right)$ extends by continuity to a unique continuous linear form still denoted by $\mathfrak{M}$, on $\mathcal{B}_{A P}^{\prime}\left(\mathbb{R}^{N}\right)$.

Proof. The mapping $\mathfrak{M}$ being continuous on $A P^{\infty}\left(\mathbb{R}^{N}\right)$ (endowed this time with the relative topology on $\left.\mathcal{B}_{\infty}^{\prime}\left(\mathbb{R}^{N}\right)\right)$, the result is therefore an immediate consequence of the density of $A P^{\infty}\left(\mathbb{R}^{N}\right)$ in $\mathcal{B}_{A P}^{\prime}\left(\mathbb{R}^{N}\right)$.

$\mathfrak{M}(T)$ is still called the mean value of $T \in \mathcal{B}_{A P}^{\prime}\left(\mathbb{R}^{N}\right)$. Now, let $T \in \mathcal{B}_{A P}^{\prime}\left(\mathbb{R}^{N}\right)$. Using the preceding lemma and the density of $A P^{\infty}\left(\mathbb{R}^{N}\right)$ in $\mathcal{B}_{A P}^{\prime}\left(\mathbb{R}^{N}\right)$, we may define the spectrum of $T$ as $\operatorname{Sp}(T)=\left\{k \in \mathbb{R}^{N}: \mathfrak{M}\left(\bar{\gamma}_{k} T\right) \neq 0\right\}$, a countable subset of $\mathbb{R}^{N}$, where $\gamma_{k}(y)=\exp (i k \cdot y)$ for $y \in \mathbb{R}^{N}$ and $\bar{\gamma}_{k}$ stands for the complex conjugation of $\gamma_{k}$. We know from [33, p.208] that if $\mathfrak{M}\left(\bar{\gamma}_{k} T\right)=0$ for any $k \in \operatorname{Sp}(T)$ then $T=0$.

Let $\mathbb{R}_{y, \tau}^{N+1}=\mathbb{R}_{y}^{N} \times \mathbb{R}_{\tau}$ denote the space $\mathbb{R}^{N} \times \mathbb{R}$ with generic variables $(y, \tau)$. It is known that $A P\left(\mathbb{R}_{y, \tau}^{N+1}\right)=A P\left(\mathbb{R}_{\tau} ; A P\left(\mathbb{R}_{y}^{N}\right)\right)$ is the closure in $\mathcal{B}\left(\mathbb{R}_{y, \tau}^{N+1}\right)$ of the tensor product $A P\left(\mathbb{R}_{y}^{N}\right) \otimes A P\left(\mathbb{R}_{\tau}\right)$ [1]. We may sometimes set $A_{y}=A P\left(\mathbb{R}_{y}^{N}\right)$, $A_{\tau}=A P\left(\mathbb{R}_{\tau}\right)$ and $A=A P\left(\mathbb{R}_{y, \tau}^{N+1}\right)$. Correspondingly, we will denote the mean value on $A_{\zeta}(\zeta=y, \tau)$ by $\mathfrak{M}_{\zeta}$.

Now let $1 \leq p<\infty$ and consider the $N$-parameter group of isometries $\{T(y)$ : $\left.y \in \mathbb{R}^{N}\right\}$ defined by

$$
T(y): \mathcal{B}_{A P}^{p}\left(\mathbb{R}^{N}\right) \rightarrow \mathcal{B}_{A P}^{p}\left(\mathbb{R}^{N}\right), T(y)(u+\mathcal{N})=u(\cdot+y)+\mathcal{N} \text { for } u \in B_{A P}^{p}\left(\mathbb{R}^{N}\right) .
$$

Since $A P\left(\mathbb{R}^{N}\right)$ consists of uniformly continuous functions, $\left\{T(y): y \in \mathbb{R}^{N}\right\}$ is a strongly continuous group in the following sense: $T(y)(u+\mathcal{N}) \rightarrow u+\mathcal{N}$ in $\mathcal{B}_{A P}^{p}\left(\mathbb{R}^{N}\right)$ as $|y| \rightarrow 0$. Using the isometric isomorphism $\mathcal{G}_{1}$ we associated to $\left\{T(y): y \in \mathbb{R}^{N}\right\}$ the following $N$-parameter group $\left\{\bar{T}(y): y \in \mathbb{R}^{N}\right\}$ defined by

$$
\begin{aligned}
& \bar{T}(y): L^{p}(\mathcal{K}) \rightarrow L^{p}(\mathcal{K}) \\
& \bar{T}(y) \mathcal{G}_{1}(u+\mathcal{N})=\mathcal{G}_{1}(T(y)(u+\mathcal{N}))=\mathcal{G}_{1}(u(\cdot+y)+\mathcal{N}) \text { for } u \in B_{A P}^{p}\left(\mathbb{R}^{N}\right) .
\end{aligned}
$$

The group $\left\{\bar{T}(y): y \in \mathbb{R}^{N}\right\}$ is also strongly continuous. The infinitesimal generator of $T(y)$ (resp. $\bar{T}(y))$ along the $i$ th coordinate direction, denoted by $D_{i, p}$ (resp. $\partial_{i, p}$ ) is defined by

$$
\begin{aligned}
& D_{i, p} u=\lim _{s \rightarrow 0} s^{-1}\left(T\left(s e_{i}\right) u-u\right) \text { in } \mathcal{B}_{A P}^{p}\left(\mathbb{R}^{N}\right) \\
& \text { (resp. } \left.\partial_{i, p} v=\lim _{s \rightarrow 0} s^{-1}\left(\bar{T}\left(s e_{i}\right) v-v\right) \text { in } L^{p}(\mathcal{K})\right),
\end{aligned}
$$

where we have used the same letter $u$ to denote the equivalence class of an element $u \in B_{A P}^{p}\left(\mathbb{R}^{N}\right)$ in $\mathcal{B}_{A P}^{p}\left(\mathbb{R}^{N}\right), e_{i}=\left(\delta_{i j}\right)_{1 \leq j \leq N}\left(\delta_{i j}\right.$ being the Kronecker $\left.\delta\right)$. The domain of $D_{i, p}\left(\operatorname{resp} . \partial_{i, p}\right)$ in $\mathcal{B}_{A P}^{p}\left(\mathbb{R}^{N}\right)$ (resp. $\left.L^{p}(\mathcal{K})\right)$ is denoted by $\mathcal{D}_{i, p}$ (resp. 
$\left.\mathcal{W}_{i, p}\right)$. It emerges from the general theory of semigroups [15, Chap. VIII, Section 1] that the following result holds.

Proposition 3. $\mathcal{D}_{i, p}\left(\right.$ resp. $\left.\mathcal{W}_{i, p}\right)$ is a vector subspace of $\mathcal{B}_{A P}^{p}\left(\mathbb{R}^{N}\right)\left(\right.$ resp. $\left.L^{p}(\mathcal{K})\right)$; $D_{i, p}: \mathcal{D}_{i, p} \rightarrow \mathcal{B}_{A P}^{p}\left(\mathbb{R}^{N}\right)$ (resp. $\partial_{i, p}: \mathcal{W}_{i, p} \rightarrow L^{p}(\mathcal{K})$ ) is a linear operator; $\mathcal{D}_{i, p}$ (resp. $\mathcal{W}_{i, p}$ ) is dense in $\mathcal{B}_{A P}^{p}\left(\mathbb{R}^{N}\right)$ (resp. $L^{p}(\mathcal{K})$ ), and the graph of $D_{i, p}\left(\right.$ resp. $\partial_{i, p}$ ) is closed in $\mathcal{B}_{A P}^{p}\left(\mathbb{R}^{N}\right) \times \mathcal{B}_{A P}^{p}\left(\mathbb{R}^{N}\right)\left(\right.$ resp. $\left.L^{p}(\mathcal{K}) \times L^{p}(\mathcal{K})\right)$.

In the sequel we denote by $\varrho$ the canonical mapping of $B_{A P}^{p}\left(\mathbb{R}^{N}\right)$ onto $\mathcal{B}_{A P}^{p}\left(\mathbb{R}^{N}\right)$, that is, $\varrho(u)=u+\mathcal{N}$ for $u \in B_{A P}^{p}\left(\mathbb{R}^{N}\right)$. The following properties are immediate. The verification can be found either in [39, Chap. B1] or in [4].

Lemma 5. Let $1 \leq i \leq N$. (1) If $u \in A P^{1}\left(\mathbb{R}^{N}\right)$ then $\varrho(u) \in \mathcal{D}_{i, p}$ and

$$
D_{i, p} \varrho(u)=\varrho\left(\frac{\partial u}{\partial y_{i}}\right) .
$$

(2) If $u \in \mathcal{D}_{i, p}$ then $\mathcal{G}_{1}(u) \in \mathcal{W}_{i, p}$ and $\mathcal{G}_{1}\left(D_{i, p} u\right)=\partial_{i, p} \mathcal{G}_{1}(u)$.

We define the higher order derivatives as follows: $D_{p}^{\alpha}=D_{1, p}^{\alpha_{1}} \circ \cdots \circ D_{N, p}^{\alpha_{N}}$ (resp. $\left.\partial_{p}^{\alpha}=\partial_{1, p}^{\alpha_{1}} \circ \cdots \circ \partial_{N, p}^{\alpha_{N}}\right)$ for $\alpha=\left(\alpha_{1}, \ldots, \alpha_{N}\right) \in \mathbb{N}^{N}$ with $D_{i, p}^{\alpha_{i}}=D_{i, p} \circ \cdots \circ D_{i, p}$, $\alpha_{i}$-times. Now, set

$$
\mathcal{B}_{A P}^{1, p}\left(\mathbb{R}^{N}\right)=\cap_{i=1}^{N} \mathcal{D}_{i, p}=\left\{u \in \mathcal{B}_{A P}^{p}\left(\mathbb{R}^{N}\right): D_{i, p} u \in \mathcal{B}_{A P}^{p}\left(\mathbb{R}^{N}\right) \forall 1 \leq i \leq N\right\}
$$

and

$$
\mathcal{D}_{A P}\left(\mathbb{R}^{N}\right)=\left\{u \in \mathcal{B}_{A P}^{\infty}\left(\mathbb{R}^{N}\right): D_{\infty}^{\alpha} u \in \mathcal{B}_{A P}^{\infty}\left(\mathbb{R}^{N}\right) \forall \alpha \in \mathbb{N}^{N}\right\}
$$

One can show that $\mathcal{D}_{A P}\left(\mathbb{R}^{N}\right)$ is dense in $\mathcal{B}_{A P}^{p}\left(\mathbb{R}^{N}\right), 1 \leq p<\infty$. Equipped with the norm

$$
\|u\|_{\mathcal{B}_{A P}^{1, p}\left(\mathbb{R}^{N}\right)}=\left(\|u\|_{p}^{p}+\sum_{i=1}^{N}\left\|D_{i, p} u\right\|_{p}^{p}\right)^{1 / p} \quad\left(u \in \mathcal{B}_{A P}^{1, p}\left(\mathbb{R}^{N}\right)\right),
$$

$\mathcal{B}_{A P}^{1, p}\left(\mathbb{R}^{N}\right)$ is a Banach space: this comes from the fact that the graph of $D_{i, p}$ is closed.

The counter-part of the above properties also holds with

$$
W^{1, p}(\mathcal{K})=\cap_{i=1}^{N} \mathcal{W}_{i, p} \text { in place of } \mathcal{B}_{A P}^{1, p}\left(\mathbb{R}^{N}\right)
$$

and

$$
\mathcal{D}(\mathcal{K})=\left\{u \in L^{\infty}(\mathcal{K}): \partial_{\infty}^{\alpha} u \in L^{\infty}(\mathcal{K}) \forall \alpha \in \mathbb{N}^{N}\right\} \text { in that of } \mathcal{D}_{A P}\left(\mathbb{R}^{N}\right) .
$$

Moreover the restriction of $\mathcal{G}_{1}$ to $\mathcal{B}_{A P}^{1, p}\left(\mathbb{R}^{N}\right)$ is an isometric isomorphism of $\mathcal{B}_{A P}^{1, p}\left(\mathbb{R}^{N}\right)$ onto $W^{1, p}(\mathcal{K})$; this is a consequence of [Part (2) of] Lemma 5 ,

Let $u \in \mathcal{D}_{i, p}(p \geq 1,1 \leq i \leq N)$. Then the inequality

$$
\left\|s^{-1}\left(T\left(s e_{i}\right) u-u\right)-D_{i, p} u\right\|_{1} \leq c\left\|s^{-1}\left(T\left(s e_{i}\right) u-u\right)-D_{i, p} u\right\|_{p}
$$

for a positive constant $c$ independent of $u$ and $s$ yields $D_{i, 1} u=D_{i, p} u$, so that $D_{i, p}$ is the restriction to $\mathcal{B}_{A P}^{p}\left(\mathbb{R}^{N}\right)$ of $D_{i, 1}$. Thus for all $u \in \mathcal{D}_{i, \infty}$ we have $u \in \mathcal{D}_{i, p}$ $(p \geq 1)$ and $D_{i, \infty} u=D_{i, p} u$ for any $1 \leq i \leq N$. The next result will be useful in the sequel.

Lemma $6\left(\right.$ [29, Lemma 2]). We have $\mathcal{D}_{A P}\left(\mathbb{R}^{N}\right)=\varrho\left(A P^{\infty}\left(\mathbb{R}^{N}\right)\right)$. 
From now on, we write $\widehat{u}$ either for $\mathcal{G}(u)$ if $u \in B_{A P}^{p}\left(\mathbb{R}^{N}\right)$ or for $\mathcal{G}_{1}(u)$ if $u \in$ $\mathcal{B}_{A P}^{p}\left(\mathbb{R}^{N}\right)$. The following properties are easily verified (see once again either [39, Chap. B1] or [4]).

Proposition 4. The following assertions hold.

(i) $\int_{\mathcal{K}} \partial_{\infty}^{\alpha} \widehat{u} d \beta=0$ for all $u \in \mathcal{D}_{A P}\left(\mathbb{R}^{N}\right)$ and $\alpha \in \mathbb{N}^{N}$;

(ii) $\int_{\mathcal{K}} \partial_{i, p} \widehat{u} d \beta=0$ for all $u \in \mathcal{D}_{i, p}$ and $1 \leq i \leq N$;

(iii) $D_{i, p}(u \phi)=u D_{i, \infty} \phi+\phi D_{i, p} u$ for all $(\phi, u) \in \mathcal{D}_{A P}\left(\mathbb{R}^{N}\right) \times \mathcal{D}_{i, p}$ and $1 \leq i \leq$ $N$.

From (iii) in Proposition 4 we have

$$
\int_{\mathcal{K}} \widehat{\phi} \partial_{i, p} \widehat{u} d \beta=-\int_{\mathcal{K}} \widehat{u} \partial_{i, \infty} \widehat{\phi} d \beta \text { for all }(u, \phi) \in \mathcal{D}_{i, p} \times \mathcal{D}_{A P}\left(\mathbb{R}^{N}\right) \text {. }
$$

This suggests us to define the notion of distributions on $\mathcal{D}_{A P}\left(\mathbb{R}^{N}\right)$ and of a weak derivative. First, let us endow $\mathcal{D}_{A P}\left(\mathbb{R}^{N}\right)=\varrho\left(A P^{\infty}\left(\mathbb{R}^{N}\right)\right)$ with its natural topology defined by the family of norms $N_{n}(u)=\sup _{|\alpha| \leq n} \sup _{y \in \mathbb{R}^{N}}\left|D_{\infty}^{\alpha} u(y)\right|$, integers $n \geq 0$. So topologized, $\mathcal{D}_{A P}\left(\mathbb{R}^{N}\right)$ is a Fréchet space. We denote by $\mathcal{D}_{A P}^{\prime}\left(\mathbb{R}^{N}\right)$ the topological dual of $\mathcal{D}_{A P}\left(\mathbb{R}^{N}\right)$ and endow it with the strong dual topology. The elements of $\mathcal{D}_{A P}^{\prime}\left(\mathbb{R}^{N}\right)$ are called the distributions on $\mathcal{D}_{A P}\left(\mathbb{R}^{N}\right)$. There exists a relationship between the space of almost periodic distributions $\mathcal{B}_{A P}^{\prime}\left(\mathbb{R}^{N}\right)$ and $\mathcal{D}_{A P}^{\prime}\left(\mathbb{R}^{N}\right)$. To be more precise, we have the following result which allows us to view $\mathcal{B}_{A P}^{\prime}\left(\mathbb{R}^{N}\right)$ as a proper subspace of $\mathcal{D}_{A P}^{\prime}\left(\mathbb{R}^{N}\right)$.

Proposition 5. Given any $T \in \mathcal{B}_{A P}^{\prime}\left(\mathbb{R}^{N}\right)$, the linear form $\Phi(T): \mathcal{D}_{A P}\left(\mathbb{R}^{N}\right) \rightarrow \mathbb{C}$ given by

$$
\langle\Phi(T), \varrho(\varphi)\rangle=\mathfrak{M}(\varphi T) \quad\left(\varphi \in A P^{\infty}\left(\mathbb{R}^{N}\right)\right)
$$

is a distribution on $\mathcal{D}_{A P}\left(\mathbb{R}^{N}\right)$. The map $\Phi: T \mapsto \Phi(T)$ is a linear continuous embedding of $\mathcal{B}_{A P}^{\prime}\left(\mathbb{R}^{N}\right)$ into $\mathcal{D}_{A P}^{\prime}\left(\mathbb{R}^{N}\right)$.

Proof. Let $\left(\varrho\left(\varphi_{j}\right)\right)_{j}$ be a sequence of functions in $\mathcal{D}_{A P}\left(\mathbb{R}^{N}\right)$ such that $\varrho\left(\varphi_{j}\right) \rightarrow 0$ in $\mathcal{D}_{A P}\left(\mathbb{R}^{N}\right)$; then $\varphi_{j} \rightarrow 0$ in $A P^{\infty}\left(\mathbb{R}^{N}\right)$. In fact assuming $\varrho\left(\varphi_{j}\right) \rightarrow 0$ in $\mathcal{D}_{A P}\left(\mathbb{R}^{N}\right)$ leads to $\mathcal{G}_{1}\left(\varrho\left(D_{y}^{\alpha} \varphi_{j}\right)\right) \equiv \mathcal{G}\left(D_{y}^{\alpha} \varphi_{j}\right) \rightarrow 0$ in $\mathcal{C}(\mathcal{K})$ for any $\alpha \in \mathbb{N}^{N}$, so that, as $\left\|D_{y}^{\alpha} \varphi_{j}\right\|_{\infty}=\left\|\mathcal{G}\left(D_{y}^{\alpha} \varphi_{j}\right)\right\|_{\infty}$ (see Theorem 2) our claim is justified. It follows that $\varphi_{j} \rightarrow 0$ in $\mathcal{B}_{A P}^{\prime}\left(\mathbb{R}^{N}\right)$, hence $\varphi_{j} T \rightarrow 0$ in $\mathcal{B}_{A P}^{\prime}\left(\mathbb{R}^{N}\right)$. We deduce from Lemma 4 that $\mathfrak{M}\left(\varphi_{j} T\right) \rightarrow 0$, from which the continuity of $\Phi(T)$ on $\mathcal{D}_{A P}\left(\mathbb{R}^{N}\right)$. Moreover, if $\Phi(T)=0$ then $\mathfrak{M}(\varphi T)=0$ for all $\varphi \in A P^{\infty}\left(\mathbb{R}^{N}\right)$, hence $\mathfrak{M}\left(\bar{\gamma}_{k} T\right)=0$ for any $k \in \mathbb{R}^{N}$. This yields $T=0$ (see [33, p.208]). $\Phi$ is then injective. It remains to check that $\Phi$ is continuous. But this follows at once by the continuity of the mean value $\mathfrak{M}$ on $\mathcal{B}_{A P}^{\prime}\left(\mathbb{R}^{N}\right)$ (see Lemma 4).

Remark 1. The map $\Phi$ defined above is not surjective since $\mathcal{B}_{A P}^{\prime}\left(\mathbb{R}^{N}\right)$ is not the topological dual of $A P^{\infty}\left(\mathbb{R}^{N}\right)$ (see 33 , p.207]). Since it is injective and continuous, $\mathcal{B}_{A P}^{\prime}\left(\mathbb{R}^{N}\right)$ can be viewed as a proper subspace of $\mathcal{D}_{A P}^{\prime}\left(\mathbb{R}^{N}\right)$.

The weak derivative of $u \in \mathcal{D}_{A P}^{\prime}\left(\mathbb{R}^{N}\right)$ is defined as follows: for any $\alpha \in \mathbb{N}^{N}, D^{\alpha} u$ stands for the distribution defined by the formula

$$
\left\langle D^{\alpha} u, \phi\right\rangle=(-1)^{|\alpha|}\left\langle u, D_{\infty}^{\alpha} \phi\right\rangle \text { for all } \phi \in \mathcal{D}_{A P}\left(\mathbb{R}^{N}\right)
$$


The space $\mathcal{D}_{A P}\left(\mathbb{R}^{N}\right)$ being dense in $\mathcal{B}_{A P}^{p}\left(\mathbb{R}^{N}\right)(1 \leq p<\infty)$, it follows that $\mathcal{B}_{A P}^{p}\left(\mathbb{R}^{N}\right) \subset \mathcal{D}_{A P}^{\prime}\left(\mathbb{R}^{N}\right)$ with continuous embedding, so that the weak derivative of any $f \in \mathcal{B}_{A P}^{p}\left(\mathbb{R}^{N}\right)$ is well defined and verifies the following functional equation:

$$
\left\langle D^{\alpha} f, \phi\right\rangle=(-1)^{|\alpha|} \int_{\mathcal{K}} \widehat{f} \partial_{\infty}^{\alpha} \widehat{\phi} d \beta \text { for all } \phi \in \mathcal{D}_{A P}\left(\mathbb{R}^{N}\right)
$$

As a special case, for $f \in \mathcal{D}_{i, p}$ we have

$$
-\int_{\mathcal{K}} \widehat{f} \partial_{i, p} \widehat{\phi} d \beta=\int_{\mathcal{K}} \widehat{\phi} \partial_{i, p} \widehat{f} d \beta \quad \forall \phi \in \mathcal{D}_{A P}\left(\mathbb{R}^{N}\right) .
$$

Hence we may identify $D_{i, p} f$ with $D^{\alpha_{i}} f, \alpha_{i}=\left(\delta_{i j}\right)_{1 \leq j \leq N}$. Conversely, if $f \in$ $\mathcal{B}_{A P}^{p}\left(\mathbb{R}^{N}\right)$ is such that there exists $f_{i} \in \mathcal{B}_{A P}^{p}\left(\mathbb{R}^{N}\right)$ with $\left\langle D^{\alpha_{i}} f, \phi\right\rangle=-\int_{\mathcal{K}} \widehat{f}_{i} \widehat{\phi} d \beta$ for all $\phi \in \mathcal{D}_{A P}\left(\mathbb{R}^{N}\right)$, then $f \in \mathcal{D}_{i, p}$ and $D_{i, p} f=f_{i}$. This allows us to justify the fact that $\mathcal{B}_{A P}^{1, p}\left(\mathbb{R}^{N}\right)$ is a Banach space under the norm $\|\cdot\|_{\mathcal{B}_{A P}^{1, p}\left(\mathbb{R}^{N}\right)}$. The same is true for $W^{1, p}(\mathcal{K})$. Moreover $\mathcal{D}_{A P}\left(\mathbb{R}^{N}\right)($ resp. $\mathcal{D}(\mathcal{K}))$ is a dense subspace of $\mathcal{B}_{A P}^{1, p}\left(\mathbb{R}^{N}\right)$ (resp. $\left.W^{1, p}(\mathcal{K})\right)$.

We now define the appropriate space of correctors. For that, let $\rho \in B_{A P}^{2}\left(\mathbb{R}^{N}\right) \cap$ $L^{\infty}\left(\mathbb{R}^{N}\right)$ be freely fixed with $\mathfrak{M}(\rho)>0(\mathfrak{M}(\rho)$ the mean value of $\rho)$. We begin by defining the following space:

$$
\mathcal{B}_{A P, \rho}^{1, p}\left(\mathbb{R}^{N}\right)=\left\{u \in \mathcal{B}_{A P}^{1, p}\left(\mathbb{R}^{N}\right): \mathfrak{M}(\rho u)=0\right\} .
$$

We endow it with the seminorm

$$
\|u\|_{\#, p}=\left(\sum_{i=1}^{N}\left\|D_{i, p} u\right\|_{p}^{p}\right)^{1 / p} \quad\left(u \in \mathcal{B}_{A P, \rho}^{1, p}\left(\mathbb{R}^{N}\right)\right) .
$$

Since $\mathfrak{M}(\rho) \neq 0$ it follows that $\|\cdot\|_{\#, p}$ is actually a norm on $\mathcal{B}_{A P, \rho}^{1, p}\left(\mathbb{R}^{N}\right)$. Under this norm $\mathcal{B}_{A P, \rho}^{1, p}\left(\mathbb{R}^{N}\right)$ is unfortunately not complete. We denote by $\mathcal{B}_{\# A P}^{1, p}\left(\mathbb{R}^{N}\right)$ its completion with respect to the above norm and by $J_{p}$ the canonical embedding of $\mathcal{B}_{A P, \rho}^{1, p}\left(\mathbb{R}^{N}\right)$ into $\mathcal{B}_{\# A P}^{1, p}\left(\mathbb{R}^{N}\right)$. It can be easily checked that $\mathcal{D}_{A P, \rho}\left(\mathbb{R}^{N}\right)=\{u \in$ $\left.\mathcal{D}_{A P}\left(\mathbb{R}^{N}\right): \mathfrak{M}(\rho u)=0\right\}$ is dense in $\mathcal{B}_{A P, \rho}^{1, p}\left(\mathbb{R}^{N}\right)$. It holds that:

$\left(\mathrm{P}_{1}\right)$ The gradient operator $D_{p}=\left(D_{1, p}, \ldots, D_{N, p}\right): \mathcal{B}_{A P, \rho}^{1, p}\left(\mathbb{R}^{N}\right) \rightarrow\left(\mathcal{B}_{A P}^{p}\left(\mathbb{R}^{N}\right)\right)^{N}$ extends by continuity to a unique mapping $\bar{D}_{p}: \mathcal{B}_{\# A P}^{1, p}\left(\mathbb{R}^{N}\right) \rightarrow\left(\mathcal{B}_{A P}^{p}\left(\mathbb{R}^{N}\right)\right)^{N}$ with the properties

$$
D_{i, p}=\bar{D}_{i, p} \circ J_{p}
$$

and

$$
\|u\|_{\#, p}=\left(\sum_{i=1}^{N}\left\|\bar{D}_{i, p} u\right\|_{p}^{p}\right)^{1 / p} \quad \text { for } u \in \mathcal{B}_{\# A P}^{1, p}\left(\mathbb{R}^{N}\right) .
$$

$\left(\mathrm{P}_{2}\right)$ The space $J_{p}\left(\mathcal{B}_{A P, \rho}^{1, p}\left(\mathbb{R}^{N}\right)\right)$ (and hence $\left.J_{p}\left(\mathcal{D}_{A P, \rho}\left(\mathbb{R}^{N}\right)\right)\right)$ is dense in $\mathcal{B}_{\# A P}^{1, p}\left(\mathbb{R}^{N}\right)$. Moreover the mapping $\bar{D}_{p}$ is an isometric embedding of $\mathcal{B}_{\# A P}^{1, p}\left(\mathbb{R}^{N}\right)$ onto a closed subspace of $\left(\mathcal{B}_{A P}^{p}\left(\mathbb{R}^{N}\right)\right)^{N}$, so that $\mathcal{B}_{\# A P}^{1, p}\left(\mathbb{R}^{N}\right)$ is a reflexive Banach space. By duality we define the divergence operator $\operatorname{div}_{p^{\prime}}:\left(\mathcal{B}_{A P}^{p}\left(\mathbb{R}^{N}\right)\right)^{N} \rightarrow\left(\mathcal{B}_{\# A P}^{1, p}\left(\mathbb{R}^{N}\right)\right)^{\prime}$ $\left(p^{\prime}=p /(p-1)\right)$ by

$$
\left\langle\operatorname{div}_{p^{\prime}} u, v\right\rangle=-\left\langle u, \bar{D}_{p} v\right\rangle \text { for } v \in \mathcal{B}_{\# A P}^{1, p}\left(\mathbb{R}^{N}\right) \text { and } u=\left(u_{i}\right) \in\left(\mathcal{B}_{A P}^{p^{\prime}}\left(\mathbb{R}^{N}\right)\right)^{N}
$$


where $\left\langle u, \bar{D}_{p} v\right\rangle=\sum_{i=1}^{N} \int_{\mathcal{K}} \widehat{u}_{i} \partial_{i, p} \widehat{v} d \beta$. The just defined $\operatorname{div}_{p^{\prime}}$ operator extends the natural divergence operator defined in $\mathcal{D}_{A P}\left(\mathbb{R}^{N}\right)$ since $D_{i, p} f=\bar{D}_{i, p}\left(J_{p} f\right)$ for all $f \in \mathcal{D}_{A P}\left(\mathbb{R}^{N}\right)$.

Now taking in (3.2) $u=D_{p^{\prime}} w$ with $w \in \mathcal{B}_{A P}^{p^{\prime}}\left(\mathbb{R}^{N}\right)$ being such that $D_{p^{\prime}} w \in$ $\left(\mathcal{B}_{A P}^{p^{\prime}}\left(\mathbb{R}^{N}\right)\right)^{N}$, we define the Laplacian operator on $\mathcal{B}_{A P}^{p^{\prime}}\left(\mathbb{R}^{N}\right)$ (denoted here by $\Delta_{p^{\prime}}$ ) as follows:

$$
\left\langle\Delta_{p^{\prime}} w, v\right\rangle=\left\langle\operatorname{div}_{p^{\prime}}\left(D_{p^{\prime}} w\right), v\right\rangle=-\left\langle D_{p^{\prime}} w, \bar{D}_{p} v\right\rangle \text { for all } v \in \mathcal{B}_{\# A P}^{1, p}\left(\mathbb{R}^{N}\right) .
$$

If in addition $v=J_{p}(\phi)$ with $\phi \in \mathcal{D}_{A P}\left(\mathbb{R}^{N}\right) / \mathbb{C}$ then $\left\langle\Delta_{p^{\prime}} w, J_{p}(\phi)\right\rangle=-\left\langle D_{p^{\prime}} w, D_{p} \phi\right\rangle$, so that, for $p=2$, we get

$$
\left\langle\Delta_{2} w, J_{2}(\phi)\right\rangle=\left\langle w, \Delta_{2} \phi\right\rangle \text { for all } w \in \mathcal{B}_{A P}^{2}\left(\mathbb{R}^{N}\right) \text { and } \phi \in \mathcal{D}_{A P}\left(\mathbb{R}^{N}\right) / \mathbb{C} .
$$

The following result is also immediate.

Proposition 6. For $u \in A P^{\infty}\left(\mathbb{R}^{N}\right)$ we have

$$
\Delta_{p} \varrho(u)=\varrho\left(\Delta_{y} u\right)
$$

where $\Delta_{y}$ stands for the usual Laplacian operator on $\mathbb{R}_{y}^{N}$.

We end this subsection with some notations. Let $f \in \mathcal{B}_{A P}^{p}\left(\mathbb{R}^{N}\right)$. We know that $D^{\alpha_{i}} f$ exists (in the sense of distributions) and that $D^{\alpha_{i}} f=D_{i, p} f$ if $f \in \mathcal{D}_{i, p}$. So we can drop the subscript $p$ and henceforth denote $D_{i, p}\left(\right.$ resp. $\partial_{i, p}$ ) by $\bar{\partial} / \partial y_{i}$ (resp. $\left.\partial_{i}\right)$. Thus, $\bar{D}_{y}$ will stand for the gradient operator $\left(\bar{\partial} / \partial y_{i}\right)_{1 \leq i \leq N}$ and $\overline{\operatorname{div}}_{y}$ for the divergence operator $\operatorname{div}_{p}$. We will also denote the operator $\bar{D}_{i, p}$ by $\bar{\partial} / \partial y_{i}$. Since $J_{p}$ is an embedding, this allows us to view $\mathcal{B}_{A P, \rho}^{1, p}\left(\mathbb{R}^{N}\right)\left(\right.$ and hence $\left.\mathcal{D}_{A P, \rho}\left(\mathbb{R}^{N}\right)\right)$ as a dense subspace of $\mathcal{B}_{\# A P}^{1, p}\left(\mathbb{R}^{N}\right)$. $D_{i, p}$ will therefore be seen as the restriction of $\bar{D}_{i, p}$ to $\mathcal{B}_{A P, \rho}^{1, p}\left(\mathbb{R}^{N}\right)$. Thus we will henceforth omit $J_{p}$ in the notation if it is understood from the context and there is no risk of confusion. This will lead to the notation $\bar{D}_{p}=\bar{D}_{y}=\left(\bar{\partial} / \partial y_{i}\right)_{1 \leq i \leq N}$ and $\partial_{p}=\partial=\left(\partial_{i}\right)_{1 \leq i \leq N}$. Finally, we will denote the Laplacian operator on $\mathcal{B}_{A P}^{p}\left(\mathbb{R}^{N}\right)$ by $\bar{\Delta}_{y}$.

3.2. The $\Sigma$-convergence. Let $A_{y}=A P\left(\mathbb{R}_{y}^{N}\right)$ and $A_{\tau}=A P\left(\mathbb{R}_{\tau}\right)$. We know that $A=A P\left(\mathbb{R}_{y, \tau}^{N+1}\right)$ is the closure in $\mathcal{B}\left(\mathbb{R}_{y, \tau}^{N+1}\right)$ of the tensor product $A_{y} \otimes A_{\tau}$. We denote by $\mathcal{K}_{y}\left(\right.$ resp. $\left.\mathcal{K}_{\tau}, \mathcal{K}\right)$ the spectrum of $A_{y}$ (resp. $\left.A_{\tau}, A\right)$. The same letter $\mathcal{G}$ will denote the Gelfand transformation on $A_{y}, A_{\tau}$ and $A$, as well. Points in $\mathcal{K}_{y}$ (resp. $\mathcal{K}_{\tau}$ ) are denoted by $s$ (resp. $s_{0}$ ). The Haar measure on the compact group $\mathcal{K}_{y}$ (resp. $\mathcal{K}_{\tau}$ ) is denoted by $\beta_{y}$ (resp. $\beta_{\tau}$ ). We have $\mathcal{K}=\mathcal{K}_{y} \times \mathcal{K}_{\tau}$ (Cartesian product) and the Haar measure on $\mathcal{K}$ is precisely the product measure $\beta=\beta_{y} \otimes \beta_{\tau}$; the last equality follows in an obvious way by the density of $A_{y} \otimes A_{\tau}$ in $A$ and by the Fubini's theorem. Finally, the letter $E$ will throughout denote $\left(\varepsilon_{n}\right)_{n \in \mathbb{N}}$ with $0<\varepsilon_{n} \leq 1$ and such that $\varepsilon_{n} \rightarrow 0$ as $n \rightarrow \infty$. In what follows, we use the same notation as in the preceding section.

Definition 1. A sequence $\left(u_{\varepsilon}\right)_{\varepsilon>0} \subset L^{p}\left(Q_{T}\right)(1 \leq p<\infty)$ is said to weakly $\Sigma$ converge in $L^{p}\left(Q_{T}\right)$ to some $u_{0} \in L^{p}\left(Q_{T} ; \mathcal{B}_{A P}^{p}\left(\mathbb{R}_{y, \tau}^{N+1}\right)\right)$ if as $\varepsilon \rightarrow 0$, we have

$$
\begin{aligned}
\int_{Q_{T}} u_{\varepsilon}(x, t) & f\left(x, t, \frac{x}{\varepsilon}, \frac{t}{\varepsilon^{2}}\right) d x d t \\
& \rightarrow \iint_{Q_{T} \times \mathcal{K}} \widehat{u}_{0}\left(x, t, s, s_{0}\right) \widehat{f}\left(x, t, s, s_{0}\right) d x d t d \beta
\end{aligned}
$$

for every $f \in L^{p^{\prime}}\left(Q_{T} ; A\right)\left(1 / p^{\prime}=1-1 / p\right)$, where $\widehat{u}_{0}=\mathcal{G}_{1} \circ u_{0}$ and $\widehat{f}=\mathcal{G}_{1} \circ(\varrho \circ f)=$ $\mathcal{G} \circ f$. We express this by writing $u_{\varepsilon} \rightarrow u_{0}$ in $L^{p}\left(Q_{T}\right)$-weak $\Sigma$. 
We recall some important results whose proofs can be founded in 24, Theorems 3.1 and 3.5] (see also [28, Theorems 3.1 and 3.6]).

Theorem 3. Let $1<p<\infty$. Let $\left(u_{\varepsilon}\right)_{\varepsilon \in E}$ be a bounded sequence in $L^{p}\left(Q_{T}\right)$. Then there exists a subsequence $E^{\prime}$ from $E$ such that the sequence $\left(u_{\varepsilon}\right)_{\varepsilon \in E^{\prime}}$ is weakly $\Sigma$-convergent in $L^{p}\left(Q_{T}\right)$.

The next result is of capital interest in the homogenization process.

Theorem 4. Let $1<p<\infty$. Let $\left(u_{\varepsilon}\right)_{\varepsilon \in E}$ be a bounded sequence in $L^{p}\left(0, T ; W_{0}^{1, p}(Q)\right)$. Then there exist a subsequence $E^{\prime}$ of $E$ and a couple of functions

$$
\left(u_{0}, u_{1}\right) \in L^{p}\left(0, T ; W_{0}^{1, p}(Q)\right) \times L^{p}\left(Q_{T} ; \mathcal{B}_{A P}^{p}\left(\mathbb{R}_{\tau} ; \mathcal{B}_{\# A P}^{1, p}\left(\mathbb{R}_{y}^{N}\right)\right)\right)
$$

such that, as $E^{\prime} \ni \varepsilon \rightarrow 0$,

$$
\begin{gathered}
u_{\varepsilon} \rightarrow u_{0} \text { in } L^{p}\left(0, T ; W_{0}^{1, p}(Q)\right) \text {-weak; } \\
\frac{\partial u_{\varepsilon}}{\partial x_{i}} \rightarrow \frac{\partial u_{0}}{\partial x_{i}}+\frac{\bar{\partial} u_{1}}{\partial y_{i}} \text { in } L^{p}\left(Q_{T}\right) \text {-weak } \Sigma, 1 \leq i \leq N .
\end{gathered}
$$

We shall also deal with the product of sequences. In this respect, we give a further

Definition 2. A sequence $\left(u_{\varepsilon}\right)_{\varepsilon>0} \subset L^{p}\left(Q_{T}\right)(1 \leq p<\infty)$ is said to strongly $\Sigma$ converge in $L^{p}\left(Q_{T}\right)$ to some $u_{0} \in L^{p}\left(Q_{T} ; \mathcal{B}_{A P}^{p}\left(\mathbb{R}_{y, \tau}^{N+1}\right)\right)$ if it is weakly $\Sigma$-convergent towards $u_{0}$ and further satisfies the following condition:

$$
\left\|u_{\varepsilon}\right\|_{L^{p}\left(Q_{T}\right)} \rightarrow\left\|\widehat{u}_{0}\right\|_{L^{p}\left(Q_{T} \times \mathcal{K}\right)} .
$$

We denote this by writing $u_{\varepsilon} \rightarrow u_{0}$ in $L^{p}\left(Q_{T}\right)$-strong $\Sigma$.

This being so we have the following.

Theorem 5 ([31, Theorem 6]). Let $1<p, q<\infty$ and $r \geq 1$ be such that $1 / r=$ $1 / p+1 / q \leq 1$. Assume $\left(u_{\varepsilon}\right)_{\varepsilon \in E} \subset L^{q}\left(Q_{T}\right)$ is weakly $\Sigma$-convergent in $L^{q}\left(Q_{T}\right)$ to some $u_{0} \in L^{q}\left(Q_{T} ; \mathcal{B}_{A P}^{q}\left(\mathbb{R}_{y, \tau}^{N+1}\right)\right)$, and $\left(v_{\varepsilon}\right)_{\varepsilon \in E} \subset L^{p}\left(Q_{T}\right)$ is strongly $\Sigma$-convergent in $L^{p}\left(Q_{T}\right)$ to some $v_{0} \in L^{p}\left(Q_{T} ; \mathcal{B}_{A P}^{p}\left(\mathbb{R}_{y, \tau}^{N+1}\right)\right)$. Then the sequence $\left(u_{\varepsilon} v_{\varepsilon}\right)_{\varepsilon \in E}$ is weakly $\Sigma$-convergent in $L^{r}\left(Q_{T}\right)$ to $u_{0} v_{0}$.

As a consequence of the above theorem the following holds.

Corollary 1. Let $\left(u_{\varepsilon}\right)_{\varepsilon \in E} \subset L^{p}\left(Q_{T}\right)$ and $\left(v_{\varepsilon}\right)_{\varepsilon \in E} \subset L^{p^{\prime}}\left(Q_{T}\right) \cap L^{\infty}\left(Q_{T}\right) \quad(1<p<$ $\infty$ and $\left.p^{\prime}=p /(p-1)\right)$ be two sequences such that:

(i) $u_{\varepsilon} \rightarrow u_{0}$ in $L^{p}\left(Q_{T}\right)$-weak $\Sigma$;

(ii) $v_{\varepsilon} \rightarrow v_{0}$ in $L^{p^{\prime}}\left(Q_{T}\right)$-strong $\Sigma$;

(iii) $\left(v_{\varepsilon}\right)_{\varepsilon \in E}$ is bounded in $L^{\infty}\left(Q_{T}\right)$.

Then $u_{\varepsilon} v_{\varepsilon} \rightarrow u_{0} v_{0}$ in $L^{p}\left(Q_{T}\right)$-weak $\Sigma$.

Regarding the reiterated $\Sigma$-convergence we refer the reader to [13, 41]. 


\section{Homogenization Results}

Throughout this section we make the following assumption on the functions involved in (2.1):

(A5) Almost periodicity. We assume that the functions $a_{i j}$ and $b$ lie in $B_{A P}^{2}\left(\mathbb{R}_{y, \tau}^{N+1}\right) \cap L^{\infty}\left(\mathbb{R}_{y, \tau}^{N+1}\right)$ for all $1 \leq i, j \leq N$. We also assume that the function $\rho$ belongs to $B_{A P}^{2}\left(\mathbb{R}^{N}\right) \cap L^{\infty}\left(\mathbb{R}^{N}\right)$ with $\mathfrak{M}_{y}(\rho)>0$. Finally the function $\tau \mapsto f(\tau, r)$ lies in $\left(A P\left(\mathbb{R}_{\tau}\right)\right)^{N}$ for any $r \in \mathbb{R}^{N}$.

4.1. Preliminary results. Let $1+\frac{2 N}{N+2} \leq p<\infty$. It is a fact that the topological dual of $\mathcal{B}_{A P}^{p}\left(\mathbb{R}_{\tau} ; \mathcal{B}_{\# A P}^{1, p}\left(\mathbb{R}_{y}^{N}\right)\right)$ is $\mathcal{B}_{A P}^{p^{\prime}}\left(\mathbb{R}_{\tau} ;\left[\mathcal{B}_{\# A P}^{1, p}\left(\mathbb{R}_{y}^{N}\right)\right]^{\prime}\right)$; this can be easily seen from the fact that $\mathcal{B}_{\# A P}^{1, p}\left(\mathbb{R}_{y}^{N}\right)$ is reflexive (see Section 2) and $\mathcal{B}_{A P}^{p}\left(\mathbb{R}_{\tau} ; \mathcal{B}_{\# A P}^{1, p}\left(\mathbb{R}_{y}^{N}\right)\right.$ ) is isometrically isomorphic to $L^{p}\left(\mathcal{K}_{\tau} ; \mathcal{B}_{\# A P}^{1, p}\left(\mathbb{R}_{y}^{N}\right)\right)$. We denote by $\langle$,$\rangle (resp. [,]) the$ duality pairing between $\mathcal{B}_{\# A P}^{1, p}\left(\mathbb{R}_{y}^{N}\right)$ (resp. $\left.\mathcal{B}_{A P}^{p}\left(\mathbb{R}_{\tau} ; \mathcal{B}_{\# A P}^{1, p}\left(\mathbb{R}_{y}^{N}\right)\right)\right)$ and $\left[\mathcal{B}_{\# A P}^{1, p}\left(\mathbb{R}_{y}^{N}\right)\right]^{\prime}$ $\left(\right.$ resp. $\left.\mathcal{B}_{A P}^{p^{\prime}}\left(\mathbb{R}_{\tau} ;\left[\mathcal{B}_{\# A P}^{1, p}\left(\mathbb{R}_{y}^{N}\right)\right]^{\prime}\right)\right)$. Hence, for $u \in \mathcal{B}_{A P}^{p^{\prime}}\left(\mathbb{R}_{\tau} ;\left[\mathcal{B}_{\# A P}^{1, p}\left(\mathbb{R}_{y}^{N}\right)\right]^{\prime}\right)$ and $v \in$ $\mathcal{B}_{A P}^{p}\left(\mathbb{R}_{\tau} ; \mathcal{B}_{\# A P}^{1, p}\left(\mathbb{R}_{y}^{N}\right)\right)$,

$$
[u, v]=\int_{\mathcal{K}_{\tau}}\left\langle\widehat{u}\left(s_{0}\right), \widehat{v}\left(s_{0}\right)\right\rangle d \beta_{\tau}\left(s_{0}\right) .
$$

For a function $\psi \in \mathcal{D}_{A P, \rho}\left(\mathbb{R}^{N}\right)$ we know that $\psi$ expresses as follows: $\psi=\varrho_{y}\left(\psi_{1}\right)$ with $\psi_{1} \in A P_{\rho}^{\infty}\left(\mathbb{R}_{y}^{N}\right)=\left\{v \in A P^{\infty}\left(\mathbb{R}_{y}^{N}\right): \mathfrak{M}\left(\rho \psi_{1}\right)=0\right\}$, where $\varrho_{y}$ denotes the canonical mapping of $B_{A P}^{p}\left(\mathbb{R}_{y}^{N}\right)$ onto $\mathcal{B}_{A P}^{p}\left(\mathbb{R}_{y}^{N}\right)$; see Section 2 . We will refer to $\psi_{1}$ as the representative of $\psi$ in $A P^{\infty}\left(\mathbb{R}_{y}^{N}\right)$. Likewise we define the representative of $\psi \in \mathcal{D}_{A P}\left(\mathbb{R}_{\tau}\right) \otimes \mathcal{D}_{A P, \rho}\left(\mathbb{R}^{N}\right)$ as an element of $A P^{\infty}\left(\mathbb{R}_{\tau}\right) \otimes A P_{\rho}^{\infty}\left(\mathbb{R}_{y}^{N}\right)$ satisfying a similar property.

With all this in mind, we have the following

Lemma 7. Let $\psi \in \mathcal{C}_{0}^{\infty}\left(Q_{T}\right) \otimes\left[\mathcal{D}_{A P}\left(\mathbb{R}_{\tau}\right) \otimes \mathcal{D}_{A P, \rho}\left(\mathbb{R}_{y}^{N}\right)\right]$ and $\psi_{1}$ be its representative in $\mathcal{C}_{0}^{\infty}\left(Q_{T}\right) \otimes\left[A P^{\infty}\left(\mathbb{R}_{\tau}\right) \otimes A P_{\rho}^{\infty}\left(\mathbb{R}_{y}^{N}\right)\right]$. Let $\left(u_{\varepsilon}\right)_{\varepsilon \in E}, E^{\prime}$ and $\left(u_{0}, u_{1}\right)$ be as in Theorem 4. Then, as $E^{\prime} \ni \varepsilon \rightarrow 0$,

$$
\int_{Q_{T}} \frac{1}{\varepsilon} u_{\varepsilon} \rho^{\varepsilon} \psi_{1}^{\varepsilon} d x d t \rightarrow \int_{Q_{T}}\left[\rho u_{1}(x, t), \psi(x, t)\right] d x d t .
$$

Proof. We recall that for $\psi_{1}$ as above, we have

$$
\psi_{1}^{\varepsilon}(x, t)=\psi_{1}\left(x, t, \frac{x}{\varepsilon}, \frac{t}{\varepsilon^{2}}\right) \text { for }(x, t) \in Q_{T} .
$$

This being so, since $\rho \psi_{1}(x, t, \cdot, \tau) \in A P_{\rho}\left(\mathbb{R}_{y}^{N}\right)=\left\{u \in A P\left(\mathbb{R}_{y}^{N}\right): \mathfrak{M}_{y}(\rho u)=0\right\}$, there exists [19] a unique $\phi \in \mathcal{C}_{0}^{\infty}\left(Q_{T}\right) \otimes\left[A P^{\infty}\left(\mathbb{R}_{\tau}\right) \otimes A P\left(\mathbb{R}_{y}^{N}\right)\right]$ such that $\rho \psi_{1}=$ $\Delta_{y} \phi$. The result follows at once by the application of [26, Lemma 3.4] (see also [12, 14] for some periodic versions of this lemma and their proofs).

For $u \in \mathcal{B}_{A P}^{p}\left(\mathbb{R}_{\tau}\right)$ we denote by $\bar{\partial} / \partial \tau$ the temporal derivative defined exactly as its spatial counterpart $\bar{\partial} / \partial y_{i}$. We also put $\partial_{0}=\mathcal{G}_{1}(\bar{\partial} / \partial \tau) . \bar{\partial} / \partial \tau$ and $\partial_{0}$ enjoy the same properties as $\bar{\partial} / \partial y_{i}$ (see Section 2). In particular, they are skew adjoint. Now, let us view $\rho \bar{\partial} / \partial \tau$ as an unbounded operator defined from $\mathcal{U}=\mathcal{B}_{A P}^{p}\left(\mathbb{R}_{\tau} ; \mathcal{B}_{\# A P}^{1, p}\left(\mathbb{R}_{y}^{N}\right)\right)$ into $\mathcal{U}^{\prime}=\mathcal{B}_{A P}^{p^{\prime}}\left(\mathbb{R}_{\tau} ;\left[\mathcal{B}_{\# A P}^{1, p}\left(\mathbb{R}_{y}^{N}\right)\right]^{\prime}\right)$. Similar to [16, pp. 1243-1244], it gives rise to an unbounded operator still denoted by $\rho \bar{\partial} / \partial \tau$ with the following properties: 
$(\mathrm{P})_{1}$ The domain of $\rho \bar{\partial} / \partial \tau$ is $\mathcal{W}=\left\{v \in \mathcal{U}: \rho \bar{\partial} v / \partial \tau \in \mathcal{U}^{\prime}\right\}$;

$(\mathrm{P})_{2} \rho \bar{\partial} / \partial \tau$ is skew adjoint, that is, for all $u, v \in \mathcal{W}$,

$$
\left[\rho \frac{\bar{\partial} v}{\partial \tau}, u\right]=-\left[\rho \frac{\bar{\partial} u}{\partial \tau}, v\right]
$$

$(\mathrm{P})_{3}$ The space $\mathcal{E}=\mathcal{D}_{A P}\left(\mathbb{R}_{\tau}\right) \otimes \mathcal{D}_{A P, \rho}\left(\mathbb{R}_{y}^{N}\right)$ is dense in $\mathcal{W}$.

The above operator will be useful in the homogenization process. This being so, the preceding lemma has an important corollary.

Corollary 2 (37, Corollary 1]). Let the hypotheses be those of Lemma 7, Assume moreover that $u_{1} \in \mathcal{W}$. Then, as $E^{\prime} \ni \varepsilon \rightarrow 0$,

$$
\int_{Q_{T}} \varepsilon u_{\varepsilon} \rho^{\varepsilon} \frac{\partial \psi_{1}^{\varepsilon}}{\partial t} d x d t \rightarrow-\int_{Q_{T}}\left[\rho \frac{\bar{\partial} u_{1}}{\partial \tau}(x, t), \psi(x, t)\right] d x d t .
$$

Now assume that $\boldsymbol{u}_{1}=\left(u_{1}^{i}\right) \in\left(\mathcal{B}_{\# A P}^{1, p}\right)^{N}$ and $\boldsymbol{\psi}=\left(\psi^{i}\right) \in\left(\mathcal{D}_{A P}\left(\mathbb{R}_{\tau}\right) \otimes \mathcal{D}_{A P, \rho}\left(\mathbb{R}_{y}^{N}\right)\right)^{N}$. Then we may still define $\left[\rho \frac{\bar{\partial} \boldsymbol{u}_{1}}{\partial \tau}(x, t), \boldsymbol{\psi}(x, t)\right]$, but this time as $\sum_{i=1}^{N}\left[\rho \frac{\bar{\partial} u_{1}^{i}}{\partial \tau}(x, t), \psi^{i}(x, t)\right]$. We will use that notation in the sequel.

We end this subsection with one further result.

Lemma 8 ([37, Lemma 5]). Let $h: \mathbb{R} \times \mathbb{R}^{N} \rightarrow \mathbb{R}^{N}$ be a continuous function verifying the following conditions:

(i) $\left|h\left(\tau, r_{1}\right)-h\left(\tau, r_{2}\right)\right| \leq k\left|r_{1}-r_{2}\right|$ for any $\tau \in \mathbb{R}$ and all $r_{1}, r_{2} \in \mathbb{R}^{N}$;

(ii) $h(\cdot, r) \in A P(\mathbb{R})$ for all $r \in \mathbb{R}^{N}$.

Let $\left(\boldsymbol{u}_{\varepsilon}\right)_{\varepsilon}$ be a sequence in $L^{2}\left(Q_{T}\right)^{N}$ such that $\boldsymbol{u}_{\varepsilon} \rightarrow \boldsymbol{u}_{0}$ in $L^{2}\left(Q_{T}\right)^{N}$ as $\varepsilon \rightarrow 0$, where $\boldsymbol{u}_{0} \in L^{2}\left(Q_{T}\right)^{N}$. Then, setting $h^{\varepsilon}\left(\boldsymbol{u}_{\varepsilon}\right)(x, t)=h\left(t / \varepsilon^{2}, \boldsymbol{u}_{\varepsilon}(x, t)\right)$ we have, as $\varepsilon \rightarrow 0$,

$$
h^{\varepsilon}\left(\boldsymbol{u}_{\varepsilon}\right) \rightarrow h\left(\cdot, \boldsymbol{u}_{0}\right) \text { in } L^{2}\left(Q_{T}\right)^{N} \text {-weak } \Sigma .
$$

4.2. Homogenization results. Before we can state the homogenization result for (2.1) we need a few notations. We begin by noting that the space $\mathcal{C}_{0}^{\infty}(0, T) \otimes \mathcal{V}$ is dense in $L^{p}(0, T ; V)$. Next, we introduce the space

$$
\mathcal{B}_{\text {div }}^{1, p}=\left\{\boldsymbol{u} \in\left(\mathcal{B}_{\# A P}^{1, p}\left(\mathbb{R}^{N}\right)\right)^{N}: \overline{\operatorname{div}}_{y} \boldsymbol{u}=0\right\}
$$

where $\overline{\operatorname{div}}_{y} \boldsymbol{u}=\sum_{i=1}^{N} \bar{\partial} u^{i} / \partial y_{i}$, and its smooth counterpart

$$
\begin{aligned}
\mathcal{V}_{\mathrm{div}} & =\left\{\boldsymbol{u} \in\left(\mathcal{D}_{A P, \rho}\left(\mathbb{R}^{N}\right)\right)^{N}: \overline{\operatorname{div}}_{y} \boldsymbol{u}=0\right\} \\
& \equiv\left\{\boldsymbol{u} \in\left(\mathcal{D}_{A P, \rho}\left(\mathbb{R}^{N}\right)\right)^{N}: \mathfrak{M}(\rho \boldsymbol{u})=0 \text { and } \overline{\operatorname{div}}_{y} \boldsymbol{u}=0\right\} .
\end{aligned}
$$

The following result holds.

Lemma 9. The space $\mathcal{V}_{\text {div }}$ is dense in $\mathcal{B}_{\text {div }}^{1, p}$.

Proof. This follows exactly in a same way as the proof of [40, Lemma 2.3].

Now, let

$$
\mathcal{W}_{\text {div }}=\left\{u \in \mathcal{B}_{A P}^{p}\left(\mathbb{R}_{\tau} ; \mathcal{B}_{\text {div }}^{1, p}\right): \rho \frac{\partial u}{\partial \tau} \in \mathcal{B}_{A P}^{p^{\prime}}\left(\mathbb{R}_{\tau} ;\left(\mathcal{B}_{\text {div }}^{1, p}\right)^{\prime}\right)\right\}
$$

We set

$$
\mathbb{F}_{0}^{1, p}=L^{p}(0, T ; V) \times L^{p}\left(Q_{T} ; \mathcal{W}_{\text {div }}\right)
$$

and

$$
\mathcal{F}_{0}^{\infty}=\left[\mathcal{C}_{0}^{\infty}(0, T) \otimes \mathcal{V}\right] \times\left[\mathcal{C}_{0}^{\infty}\left(Q_{T}\right) \otimes\left(\mathcal{D}_{A P}\left(\mathbb{R}_{\tau}\right) \otimes \mathcal{V}_{\text {div }}\right)\right]
$$


Thanks to Lemma 9 and to the density of $\mathcal{E}=\mathcal{D}_{A P}\left(\mathbb{R}_{\tau}\right) \otimes \mathcal{D}_{A P, \rho}\left(\mathbb{R}_{y}^{N}\right)$ in $\mathcal{W}$, we have the density of $\mathcal{F}_{0}^{\infty}$ in $\mathbb{F}_{0}^{1, p}$.

Let $\left(\boldsymbol{u}_{\varepsilon}\right)_{\varepsilon \in E}$ be a sequence of solution to (2.1) (or to (2.9)) where we assume that $E$ is an ordinary sequence tending to zero with $\varepsilon$. In view of Proposition 1, there are a subsequence $E^{\prime}$ of $E$ and a function $\boldsymbol{u}_{0} \in L^{2}\left(Q_{T}\right)^{N}$ such that, as $E^{\prime} \ni \varepsilon \rightarrow 0$

$$
\boldsymbol{u}_{\varepsilon} \rightarrow \boldsymbol{u}_{0} \text { in } L^{2}\left(Q_{T}\right)^{N} \text {. }
$$

In view of (2.11) and by the diagonal process, one can find a subsequence of $\left(\boldsymbol{u}_{\varepsilon}\right)_{\varepsilon \in E^{\prime}}$ (not relabeled) which weakly converges in $L^{p}(0, T ; V)$ to the function $\boldsymbol{u}_{0}$ (this means that $\left.\boldsymbol{u}_{0} \in L^{p}(0, T ; V)\right)$. From Theorem 4 , we infer the existence of a function $\boldsymbol{u}_{1}=\left(u_{1}^{k}\right)_{1 \leq k \leq N} \in L^{p}\left(Q_{T} ; \mathcal{B}_{A P}^{p}\left(\mathbb{R}_{\tau} ; \mathcal{B}_{\# A P}^{1, p}\right)^{N}\right)$ such that the convergence result

$$
\frac{\partial \boldsymbol{u}_{\varepsilon}}{\partial x_{i}} \rightarrow \frac{\partial \boldsymbol{u}_{0}}{\partial x_{i}}+\frac{\bar{\partial} \boldsymbol{u}_{1}}{\partial y_{i}} \text { in } L^{p}\left(Q_{T}\right)^{N} \text {-weak } \Sigma(1 \leq i \leq N)
$$

holds when $E^{\prime} \ni \varepsilon \rightarrow 0$. Owing to Lemma 3 (see (2.16) therein) there exist a subsequence of $E^{\prime}$ (still denoted by $E^{\prime}$ ) and a function $q \in L^{p^{\prime}}\left(Q_{T} ; \mathcal{B}_{A P}^{p^{\prime}}\left(\mathbb{R}^{N+1}\right)\right.$ ) with $\int_{Q} q d x=0$ such that

$$
q_{\varepsilon} \rightarrow q \text { in } L^{p^{\prime}}\left(Q_{T}\right) \text {-weak } \Sigma \text { as } E^{\prime} \ni \varepsilon \rightarrow 0 .
$$

We recall that $\frac{\partial \boldsymbol{u}_{0}}{\partial x_{i}}=\left(\frac{\partial u_{0}^{k}}{\partial x_{i}}\right)_{1 \leq k \leq N}\left(\boldsymbol{u}_{0}=\left(u_{0}^{k}\right)_{1 \leq k \leq N}\right)$ and $\frac{\bar{\partial} \boldsymbol{u}_{1}}{\partial y_{i}}=\left(\frac{\bar{\partial} u_{1}^{k}}{\partial y_{i}}\right)_{1 \leq k \leq N}$. Now, let us consider the following functionals:

$$
\begin{aligned}
\widehat{a}_{I}(\boldsymbol{u}, \boldsymbol{v})= & \sum_{i, j, k=1}^{N} \iint_{Q_{T} \times \mathcal{K}} \widehat{a}_{i j}\left(s, s_{0}\right) \mathbb{D}_{j} u^{k} \mathbb{D}_{i} v^{k} d x d t d \beta \\
& \quad+\sum_{i, j=1}^{N} \iint_{Q_{T} \times \mathcal{K}} \widehat{b}\left(s, s_{0}\right)|\mathbb{D} \boldsymbol{u}|^{p-2} \mathbb{D}_{i} u^{j} \mathbb{D}_{i} v^{j} d x d t d \beta
\end{aligned}
$$

where $\mathbb{D}_{j} u^{k}=\frac{\partial u_{0}^{k}}{\partial x_{j}}+\partial_{j} \widehat{u}_{1}^{k}\left(\partial_{j} \widehat{u}_{1}^{k}=\mathcal{G}_{1}\left(\frac{\bar{\partial} u_{1}^{k}}{\partial y_{j}}\right)\right.$, and the same definition for $\left.\mathbb{D}_{i} v^{k}\right)$ and $\mathbb{D} \boldsymbol{u}=\left(\mathbb{D}_{j} \boldsymbol{u}\right)_{1 \leq j \leq N}$ with $\mathbb{D}_{j} \boldsymbol{u}=\left(\mathbb{D}_{j} u^{k}\right)_{1 \leq k \leq N} ;$

$$
\widehat{b}_{I}(\boldsymbol{u}, \boldsymbol{v}, \boldsymbol{w})=\sum_{i, k=1}^{N} \iint_{Q_{T}} u_{0}^{i} \frac{\partial v_{0}^{k}}{\partial x_{i}} w_{0}^{k} d x d t
$$

for $\boldsymbol{u}=\left(\boldsymbol{u}_{0}, \boldsymbol{u}_{1}\right), \boldsymbol{v}=\left(\boldsymbol{v}_{0}, \boldsymbol{v}_{1}\right), \boldsymbol{w}=\left(\boldsymbol{w}_{0}, \boldsymbol{w}_{1}\right) \in \mathbb{F}_{0}^{1, p}$. The functionals $\widehat{a}_{I}$ and $\widehat{b}_{I}$ are well-defined. Next, associated to these functionals is the variational problem

$$
\left\{\begin{array}{l}
\boldsymbol{u}=\left(\boldsymbol{u}_{0}, \boldsymbol{u}_{1}\right) \in \mathbb{F}_{0}^{1, p}: \\
-\mathfrak{M}_{y}(\rho) \int_{Q_{T}} \boldsymbol{u}_{0} \cdot \boldsymbol{\psi}_{0}^{\prime} d x d t-\int_{Q_{T}}\left[\rho \boldsymbol{u}_{1}, \frac{\partial \boldsymbol{\psi}_{1}}{\partial \tau}\right] d x d t+\widehat{a}_{I}(\boldsymbol{u}, \mathbf{\Phi})+\widehat{b}_{I}(\boldsymbol{u}, \boldsymbol{u}, \mathbf{\Phi}) \\
\quad=\int_{Q_{T}} \mathfrak{M}\left(\rho f\left(\cdot, \boldsymbol{u}_{0}\right)\right) \cdot \boldsymbol{\psi}_{0} d x d t \text { for all } \Phi=\left(\boldsymbol{\psi}_{0}, \boldsymbol{\psi}_{1}\right) \in \mathcal{F}_{0}^{\infty} .
\end{array}\right.
$$

The following global homogenization result holds.

Theorem 6. The couple $\left(\boldsymbol{u}_{0}, \boldsymbol{u}_{1}\right)$ determined by (4.1)-(4.2) solves problem (4.4).

Proof. From the equality $\operatorname{div} \boldsymbol{u}_{\varepsilon}=0$ we easily derive $\operatorname{div} \boldsymbol{u}_{0}=0$ and $\overline{\operatorname{div}}_{y} \boldsymbol{u}_{1}=0$. In order to show that $\boldsymbol{u}=\left(\boldsymbol{u}_{0}, \boldsymbol{u}_{1}\right) \in \mathbb{F}_{0}^{1, p}$ we need to verify that $\partial \boldsymbol{u}_{1}(x, t) / \partial \tau \in$ $\mathcal{B}_{A P}^{p^{\prime}}\left(\mathbb{R}_{\tau} ;\left(\mathcal{B}_{\text {div }}^{1, p}\right)^{\prime}\right)$ for a.e. $(x, t) \in Q_{T}$. But this will be done later. Now, we first need to show that $\boldsymbol{u}$ solve (4.4). For that, let $\boldsymbol{\Phi}=\left(\boldsymbol{\psi}_{0}, \boldsymbol{\psi}_{1}\right)$ with $\boldsymbol{\psi}_{0} \in \mathcal{C}_{0}^{\infty}\left(Q_{T}\right)^{N}$ 
and $\boldsymbol{\psi}_{1}=\varrho_{y}^{N}(\boldsymbol{\psi}) \equiv\left(\varrho_{y}\left(\psi_{j}\right)\right)_{1 \leq j \leq N} \in \mathcal{C}_{0}^{\infty}\left(Q_{T}\right) \otimes\left(\mathcal{D}_{A P}\left(\mathbb{R}_{\tau}\right) \otimes \mathcal{V}_{\text {div }}\right), \varrho_{y}$ being the canonical mapping from $B_{A P}^{p}\left(\mathbb{R}_{y}^{N}\right)$ into $\mathcal{B}_{A P}^{p}\left(\mathbb{R}_{y}^{N}\right)$; define $\boldsymbol{\Phi}_{\varepsilon}$ as follows:

$$
\boldsymbol{\Phi}_{\varepsilon}(x, t)=\boldsymbol{\psi}_{0}(x, t)+\varepsilon \boldsymbol{\psi}\left(x, t, \frac{x}{\varepsilon}, \frac{t}{\varepsilon^{2}}\right) \text { for }(x, t) \in Q_{T} .
$$

Then we have $\boldsymbol{\Phi}_{\varepsilon} \in \mathcal{C}_{0}^{\infty}\left(Q_{T}\right)^{N}$ and, using $\boldsymbol{\Phi}_{\varepsilon}$ as a test function in the variational formulation of (2.1) we get

$$
\begin{aligned}
& -\int_{Q_{T}} \rho^{\varepsilon} \boldsymbol{u}_{\varepsilon} \cdot \frac{\partial \boldsymbol{\Phi}_{\varepsilon}}{\partial t} d x d t+\int_{Q_{T}} a^{\varepsilon} D \boldsymbol{u}_{\varepsilon} \cdot D \boldsymbol{\Phi}_{\varepsilon} d x d t+\int_{0}^{T} b_{I}\left(\boldsymbol{u}_{\varepsilon}, \boldsymbol{u}_{\varepsilon}, \boldsymbol{\Phi}_{\varepsilon}\right) d t \\
& +\int_{Q_{T}} b^{\varepsilon}\left|D \boldsymbol{u}_{\varepsilon}\right|^{p-2} D \boldsymbol{u}_{\varepsilon} \cdot D \boldsymbol{\Phi}_{\varepsilon} d x d t-\int_{Q_{T}} q_{\varepsilon} \operatorname{div} \boldsymbol{\Phi}_{\varepsilon} d x d t \\
= & \int_{0}^{T}\left(\rho^{\varepsilon} f^{\varepsilon}\left(t, \boldsymbol{u}_{\varepsilon}(t)\right), \boldsymbol{\Phi}_{\varepsilon}\right) d t .
\end{aligned}
$$

We pass to the limit in (4.5) by considering each term separately. First we have

$$
\int_{Q_{T}} \rho^{\varepsilon} \boldsymbol{u}_{\varepsilon} \cdot \frac{\partial \Phi_{\varepsilon}}{\partial t} d x d t=\int_{Q_{T}} \rho^{\varepsilon} \boldsymbol{u}_{\varepsilon} \cdot \frac{\partial \boldsymbol{\psi}_{0}}{\partial t} d x d t+\varepsilon \int_{Q_{T}} \rho^{\varepsilon} \boldsymbol{u}_{\varepsilon} \cdot \frac{\partial \boldsymbol{\psi}^{\varepsilon}}{\partial t} d x d t
$$

In view of Corollary 2 we have that

$$
\varepsilon \int_{Q_{T}} \rho^{\varepsilon} \boldsymbol{u}_{\varepsilon} \cdot \frac{\partial \boldsymbol{\psi}^{\varepsilon}}{\partial t} d x d t \rightarrow \int_{Q_{T}}\left[\rho \boldsymbol{u}_{1}, \frac{\partial \boldsymbol{\psi}_{1}}{\partial \tau}\right] d x d t \text { when } E^{\prime} \ni \varepsilon \rightarrow 0
$$

On the other hand, due to (4.1) we get

$$
\int_{Q_{T}} \rho^{\varepsilon} \boldsymbol{u}_{\varepsilon} \cdot \frac{\partial \boldsymbol{\psi}_{0}}{\partial t} d x d t \rightarrow \mathfrak{M}_{y}(\rho) \int_{Q_{T}} \boldsymbol{u}_{0} \cdot \frac{\partial \boldsymbol{\psi}_{0}}{\partial t} d x d t \text { as } E^{\prime} \ni \varepsilon \rightarrow 0 .
$$

Hence, as $E^{\prime} \ni \varepsilon \rightarrow 0$,

$$
\int_{Q_{T}} \boldsymbol{u}_{\varepsilon} \cdot \frac{\partial \boldsymbol{\Phi}_{\varepsilon}}{\partial t} d x d t \rightarrow \mathfrak{M}_{y}(\rho) \int_{Q_{T}} \boldsymbol{u}_{0} \cdot \frac{\partial \boldsymbol{\psi}_{0}}{\partial t} d x d t+\int_{Q_{T}}\left[\rho \boldsymbol{u}_{1}, \frac{\partial \boldsymbol{\psi}_{1}}{\partial \tau}\right] d x d t
$$

Next, it is an usual well known fact that (see e.g., [13, Lemma 3.5]), the convergence result (4.2) together with the weak $\Sigma$-convergence of the sequence $\left(D \boldsymbol{\Phi}_{\varepsilon}\right)_{\varepsilon}$ to $\mathbb{D} \boldsymbol{\Phi}$, imply

$$
\int_{Q_{T}} a^{\varepsilon} D \boldsymbol{u}_{\varepsilon} \cdot D \boldsymbol{\Phi}_{\varepsilon} d x d t \rightarrow \sum_{i, j, k=1}^{N} \iint_{Q_{T} \times \mathcal{K}} \widehat{a}_{i j}\left(s, s_{0}\right) \mathbb{D}_{j} u^{k} \mathbb{D}_{i} v^{k} d x d t d \beta .
$$

Considering the next term, we use the monotonicity property to have

$$
\int_{Q_{T}}\left(b^{\varepsilon}\left|D \boldsymbol{u}_{\varepsilon}\right|^{p-2} D \boldsymbol{u}_{\varepsilon}-b^{\varepsilon}\left|D \boldsymbol{\Phi}_{\varepsilon}\right|^{p-2} D \boldsymbol{\Phi}_{\varepsilon}\right) \cdot\left(D \boldsymbol{u}_{\varepsilon}-D \boldsymbol{\Phi}_{\varepsilon}\right) d x d t \geq 0 .
$$

Owing to the estimate (2.11) we infer that

$$
\sup _{\varepsilon>0}\left\|b^{\varepsilon}\left|D \boldsymbol{u}_{\varepsilon}\right|^{p-2} D \boldsymbol{u}_{\varepsilon}\right\|_{L^{p^{\prime}}\left(Q_{T}\right)^{N \times N}}^{p^{\prime}}<\infty,
$$

so that, from Theorem 3, there exist a function $\chi \in L^{p^{\prime}}\left(Q_{T} ; \mathcal{B}_{A P}^{p^{\prime}}\left(\mathbb{R}^{N}\right)\right)^{N \times N}$ and a subsequence of $E^{\prime}$ not relabeled, such that $b^{\varepsilon}\left|D \boldsymbol{u}_{\varepsilon}\right|^{p-2} D \boldsymbol{u}_{\varepsilon} \rightarrow \chi$ in $L^{p^{\prime}}\left(Q_{T}\right)^{N \times N_{-}}$ weak $\Sigma$ as $E^{\prime} \ni \varepsilon \rightarrow 0$. We therefore argue as in the proof of [25, Theorem 3.1] (see 
also [41, Theorem 3.2]) to pass to the limit in (4.6) (as $\left.E^{\prime} \ni \varepsilon \rightarrow 0\right)$ and get

$$
\left.\iint_{Q_{T} \times \mathcal{K}}\left(\widehat{\chi}-\widehat{b}|\mathbb{D} \boldsymbol{\Phi}|^{p-2} \mathbb{D} \Phi\right)\right) \cdot(\mathbb{D} \boldsymbol{u}-\mathbb{D} \boldsymbol{\Phi}) d x d t d \beta \geq 0
$$

for any $\Phi \in \mathcal{F}_{0}^{\infty}$ where, as above, $\mathbb{D} \boldsymbol{u}=D \boldsymbol{u}_{0}+\partial \widehat{\boldsymbol{u}}_{1}\left(\boldsymbol{u}=\left(\boldsymbol{u}_{0}, \boldsymbol{u}_{1}\right)\right)$ and $\mathbb{D} \boldsymbol{\Phi}=$ $D \boldsymbol{\psi}_{0}+\partial \widehat{\boldsymbol{\psi}}_{1}$. By the density of $\mathcal{F}_{0}^{\infty}$ in $\mathbb{F}_{0}^{1, p}$ and by a continuity argument, (4.7) still holds for $\boldsymbol{\Phi} \in \mathbb{F}_{0}^{1, p}$. Hence by taking $\boldsymbol{\Phi}=\boldsymbol{u}+\lambda \boldsymbol{v}$ for $\boldsymbol{v}=\left(\boldsymbol{v}_{0}, \boldsymbol{v}_{1}\right) \in \mathbb{F}_{0}^{1, p}$ and $\lambda>0$ arbitrarily fixed, we get

$$
\lambda \iint_{Q_{T} \times \mathcal{K}}(\widehat{\chi}-\widehat{b}(\cdot, \mathbb{D} \boldsymbol{u}+\lambda \mathbb{D} \boldsymbol{v})) \cdot \mathbb{D} \boldsymbol{v} d x d t d \overline{\mathbb{P}} d \beta \geq 0 \text { for all } \boldsymbol{v} \in \mathbb{F}_{0}^{1, p} .
$$

Therefore by a mere routine, we deduce that $\chi=b\left|D \boldsymbol{u}_{0}+\bar{D}_{y} \boldsymbol{u}_{1}\right|^{p-2}\left(D \boldsymbol{u}_{0}+\bar{D}_{y} \boldsymbol{u}_{1}\right)$.

The next point to check is to compute the $\lim _{\varepsilon \rightarrow 0} \int_{0}^{T} b_{I}\left(\boldsymbol{u}_{\varepsilon}, \boldsymbol{u}_{\varepsilon}, \Phi_{\varepsilon}\right) d t$. We claim that, as $\varepsilon \rightarrow 0$,

$$
\int_{0}^{T} b_{I}\left(\boldsymbol{u}_{\varepsilon}, \boldsymbol{u}_{\varepsilon}, \Phi_{\varepsilon}\right) d t \rightarrow \widehat{b}_{I}(\boldsymbol{u}, \boldsymbol{u}, \boldsymbol{\Phi}) .
$$

Indeed, by the strong convergence result (4.1) in conjunction with the Theorem 5 , our claim is justified.

We obviously have that

$$
\begin{aligned}
\int_{0}^{T}\left(\rho^{\varepsilon} f^{\varepsilon}\left(t, \boldsymbol{u}_{\varepsilon}(t)\right), \boldsymbol{\Phi}_{\varepsilon}\right) d t= & \int_{Q_{T}} \rho^{\varepsilon} f^{\varepsilon}\left(t, \boldsymbol{u}_{\varepsilon}(t)\right) \cdot \boldsymbol{\Phi}_{\varepsilon} d x d t \\
= & \int_{Q_{T}} \rho^{\varepsilon} f^{\varepsilon}\left(t, \boldsymbol{u}_{\varepsilon}(t)\right) \cdot \boldsymbol{\psi}_{0} d x d t \\
& +\varepsilon \int_{Q_{T}} \rho^{\varepsilon} f^{\varepsilon}\left(t, \boldsymbol{u}_{\varepsilon}(t)\right) \cdot \boldsymbol{\psi}^{\varepsilon} d x d t .
\end{aligned}
$$

It can easily be seen that the second integral of the right-hand side goes to zero with $\varepsilon \in E^{\prime}$. For the first one, taking $\rho^{\varepsilon} \boldsymbol{\psi}_{0}$ as a test function and using Lemma 8 we are led to

$$
\begin{aligned}
\int_{Q_{T}} \rho^{\varepsilon} f^{\varepsilon}\left(\cdot, \boldsymbol{u}_{\varepsilon}\right) \cdot \boldsymbol{\psi}_{0} d x d t & \rightarrow \iint_{Q_{T} \times \mathcal{K}} \widehat{\rho} \widehat{f}\left(\cdot, \boldsymbol{u}_{0}\right) \cdot \boldsymbol{\psi}_{0} d x d t d \beta \\
& =\int_{Q_{T}} \mathfrak{M}\left(\rho f\left(\cdot, \boldsymbol{u}_{0}\right)\right) \cdot \boldsymbol{\psi}_{0} d x d t
\end{aligned}
$$

Now, on the basis of (4.3) there is no difficulty in showing that

$$
\int_{Q_{T}} q_{\varepsilon} \operatorname{div} \boldsymbol{\Phi}_{\varepsilon} d x d t \rightarrow \iint_{Q_{T} \times \mathcal{K}} \widehat{q} \operatorname{div} \boldsymbol{\psi}_{0} d x d t d \beta .
$$

It emerges from the above study that $\boldsymbol{u}=\left(\boldsymbol{u}_{0}, \boldsymbol{u}_{1}\right)$ and $q_{0}=\mathfrak{M}(q):=\int_{\mathcal{K}} \widehat{q} d \beta$ satisfies

$$
\begin{aligned}
& -\mathfrak{M}_{y}(\rho) \int_{Q_{T}} \boldsymbol{u}_{0} \cdot \boldsymbol{\psi}_{0}^{\prime} d x d t-\int_{Q_{T}}\left[\rho \boldsymbol{u}_{1}, \frac{\partial \boldsymbol{\psi}_{1}}{\partial \tau}\right] d x d t+\widehat{a}_{I}(\boldsymbol{u}, \mathbf{\Phi})+\widehat{b}_{I}(\boldsymbol{u}, \boldsymbol{u}, \mathbf{\Phi}) \\
& -\int_{Q_{T}} q_{0} \operatorname{div} \boldsymbol{\psi}_{0} d x d t=\int_{Q_{T}} \mathfrak{M}\left(\rho f\left(\cdot, \boldsymbol{u}_{0}\right)\right) \cdot \boldsymbol{\psi}_{0} d x d t
\end{aligned}
$$

for all $\boldsymbol{\Phi}=\left(\boldsymbol{\psi}_{0}, \boldsymbol{\psi}_{1}\right)$ as above. Choosing in particular $\boldsymbol{\Phi} \in \mathcal{F}_{0}^{\infty}$ we end up with $\left(\boldsymbol{u}_{0}, \boldsymbol{u}_{1}\right)$ solving (4.4) since $\operatorname{div} \boldsymbol{\psi}_{0}=0$ in that case. 
Problem (4.4) is the global homogenized problem. In order to derive the homogenized problem, we need to uncouple problem (4.4). As we can see, (4.4) is equivalent to the following system (4.9)-(4.10):

$$
\begin{aligned}
& \left\{\begin{array}{l}
-\int_{Q_{T}}\left[\rho \boldsymbol{u}_{1}, \frac{\partial \boldsymbol{\psi}_{1}}{\partial \tau}\right] d x d t+\iint_{Q_{T} \times \mathcal{K}} \widehat{a} \mathbb{D} \boldsymbol{u} \cdot \partial \widehat{\boldsymbol{\psi}}_{1} d x d t d \beta \\
+\iint_{Q_{T} \times \mathcal{K}} \widehat{b}|\mathbb{D} \boldsymbol{u}|^{p-2} \mathbb{D} \boldsymbol{u} \cdot \partial \widehat{\boldsymbol{\psi}}_{1} d x d t d \beta=0 \text { for all } \boldsymbol{\psi}_{1} \in \mathcal{C}_{0}^{\infty}\left(Q_{T}\right) \otimes\left[\mathcal{D}_{A_{\tau}}\left(\mathbb{R}_{\tau}\right) \otimes \mathcal{V}_{\operatorname{div}}\right]
\end{array}\right. \\
& \left\{\begin{array}{c}
-\mathfrak{M}_{y}(\rho) \int_{Q_{T}} \boldsymbol{u}_{0} \cdot \boldsymbol{\psi}_{0}^{\prime} d x d t+\widehat{a}_{I}\left(\boldsymbol{u},\left(\boldsymbol{\psi}_{0}, 0\right)\right)+\widehat{b}_{I}\left(\boldsymbol{u}, \boldsymbol{u},\left(\boldsymbol{\psi}_{0}, 0\right)\right) \\
=\int_{Q_{T}} \mathfrak{M}\left(\rho f\left(\cdot, \boldsymbol{u}_{0}\right)\right) \cdot \boldsymbol{\psi}_{0} d x d t \text { for all } \boldsymbol{\psi}_{0} \in \mathcal{C}_{0}^{\infty}(0, T) \otimes \mathcal{V}
\end{array}\right.
\end{aligned}
$$

It is an easy matter to deal with (4.9). In fact, fix $\xi \in \mathbb{R}^{N \times N}$ and consider the following cell problem:

$$
\left\{\begin{array}{l}
\boldsymbol{\pi}(\xi) \in \mathcal{B}_{A P}^{p}\left(\mathbb{R}_{\tau} ; \mathcal{B}_{\text {div }}^{1, p}\right): \\
{\left[\rho \frac{\partial \boldsymbol{\pi}}{\partial \tau}, \boldsymbol{w}\right]+\int_{\mathcal{K}} \widehat{b}|\xi+\partial \widehat{\boldsymbol{\pi}}(\xi)|^{p-2}(\xi+\partial \widehat{\boldsymbol{\pi}}(\xi)) \cdot \partial \widehat{\boldsymbol{w}} d \beta} \\
+\int_{\mathcal{K}} \widehat{a}(\xi+\partial \widehat{\boldsymbol{\pi}}(\xi)) \cdot \partial \widehat{\boldsymbol{w}} d \beta=0 \text { for all } \boldsymbol{w} \in \mathcal{B}_{A_{\tau}}^{p}\left(\mathbb{R}_{\tau} ; \mathcal{B}_{\mathrm{div}}^{1, p}\right)
\end{array}\right.
$$

Assume for a while that the solution to (4.11) exists. Then, due to the properties of the functions $a$ and $b$, the linear functional

$$
\boldsymbol{w} \mapsto \int_{\mathcal{K}} \widehat{b}|\xi+\partial \widehat{\boldsymbol{\pi}}(\xi)|^{p-2}(\xi+\partial \widehat{\boldsymbol{\pi}}(\xi)) \cdot \partial \widehat{\boldsymbol{w}} d \beta+\int_{\mathcal{K}} \widehat{a}(\xi+\partial \widehat{\boldsymbol{\pi}}(\xi)) \cdot \partial \widehat{\boldsymbol{w}} d \beta
$$

defined on $\mathcal{B}_{A P}^{p}\left(\mathbb{R}_{\tau} ; \mathcal{B}_{\text {div }}^{1, p}\right)$ is continuous, so that the linear functional $\boldsymbol{w} \mapsto\left[\rho \frac{\partial \boldsymbol{\pi}}{\partial \tau}, \boldsymbol{w}\right]$, defined on $\mathcal{E}_{\text {div }}=\mathcal{D}_{A P}\left(\mathbb{R}_{\tau}\right) \otimes \mathcal{V}_{\text {div }}$, is continuous when endowing $\mathcal{E}_{\text {div }}$ with the $\mathcal{V}$ norm. From the density of $\mathcal{E}_{\text {div }}$ in $\mathcal{B}_{A P}^{p}\left(\mathbb{R}_{\tau} ; \mathcal{B}_{\text {div }}^{1, p}\right.$ ) (see Lemma 9) we get readily $\rho \frac{\partial \pi}{\partial \tau} \in \mathcal{B}_{A P}^{p^{\prime}}\left(\mathbb{R}_{\tau} ;\left(\mathcal{B}_{\text {div }}^{1, p}\right)^{\prime}\right)$, so that $\boldsymbol{\pi}$ lies in $\mathcal{W}_{\text {div }}$. Since $\mathcal{E}_{\text {div }}$ is dense in $\mathcal{W}_{\text {div }}$, Eq. (4.11) still holds for $\boldsymbol{w} \in \mathcal{W}_{\text {div }}$. But if $\boldsymbol{\pi}_{1}=\boldsymbol{\pi}_{1}(\xi)$ and $\boldsymbol{\pi}_{2}=\boldsymbol{\pi}_{2}(\xi)$ are two solutions of (4.11) then, setting $\boldsymbol{\pi}=\boldsymbol{\pi}_{1}-\boldsymbol{\pi}_{2}$,

$$
\left\{\begin{array}{l}
\int_{\mathcal{K}} \widehat{a} \partial \widehat{\boldsymbol{\pi}} \cdot \partial \widehat{\boldsymbol{w}} d \beta+\int_{\mathcal{K}}\left(\widehat{b}\left(\cdot, \xi+\partial \widehat{\boldsymbol{\pi}}_{1}\right)-\widehat{b}\left(\cdot, \xi+\partial \widehat{\boldsymbol{\pi}}_{2}\right)\right) \cdot \partial \widehat{\boldsymbol{w}} d \beta=0 \\
\text { for all } \boldsymbol{w} \in \mathcal{W}_{\text {div }} .
\end{array}\right.
$$

Taking the particular test function $\boldsymbol{w}=\boldsymbol{\pi}$ and using the equality $\left[\rho \frac{\partial \boldsymbol{\pi}}{\partial \tau}, \boldsymbol{\pi}\right]=0$, we are led to

$$
\int_{\mathcal{K}} \widehat{a} \partial \widehat{\boldsymbol{\pi}} \cdot \partial \widehat{\boldsymbol{\pi}} d \beta+\int_{\Delta(A)}\left(\widehat{b}\left(\cdot, \xi+\partial \widehat{\boldsymbol{\pi}}_{1}\right)-\widehat{b}\left(\cdot, \xi+\partial \widehat{\boldsymbol{\pi}}_{2}\right)\right) \cdot \partial \widehat{\boldsymbol{\pi}} d \beta=0
$$

and using once again the properties of $a$ and $b$, we get

$$
\nu_{0} \int_{\mathcal{K}}|\partial \widehat{\pi}|^{2} d \beta+\nu_{1} \int_{\mathcal{K}}|\partial \widehat{\pi}|^{p} d \beta=0
$$

which gives $\partial \widehat{\boldsymbol{\pi}}=0$, or equivalently, $\bar{D}_{y} \boldsymbol{\pi}=0$. It then follows that $\boldsymbol{\pi}=0$ since it belong to $\mathcal{B}_{A P}^{p}\left(\mathbb{R}_{\tau} ;\left(\mathcal{B}_{\# A P}^{1, p}\left(\mathbb{R}_{y}^{N}\right)\right)^{N}\right)$. Returning to the existence issue, Eq. (4.11) admits a solution; see e.g., [32, Proposition III.4.1].

Now, choosing $\boldsymbol{\psi}_{1}=\varphi \otimes \boldsymbol{w}$ in (4.9) with $\varphi \in \mathcal{C}_{0}^{\infty}\left(Q_{T}\right)$ and $\boldsymbol{w} \in\left[\mathcal{D}_{A P}\left(\mathbb{R}_{\tau}\right) \otimes \mathcal{V}_{\text {div }}\right]$, and using the arbitrariness of $\varphi$ we get the following equation:

$$
-\left[\rho \boldsymbol{u}_{1}, \frac{\partial \boldsymbol{w}}{\partial \tau}\right]+\int_{\mathcal{K}} \widehat{a} \mathbb{D} \boldsymbol{u} \cdot \partial \widehat{\boldsymbol{w}} d \beta+\int_{\mathcal{K}} \widehat{b}|\mathbb{D} \boldsymbol{u}|^{p-2} \mathbb{D} \boldsymbol{u} \cdot \partial \widehat{\boldsymbol{w}} d \beta=0 \text { for all } \boldsymbol{w} \in \mathcal{D}_{A P}\left(\mathbb{R}_{\tau}\right) \otimes \mathcal{V}_{\text {div }}
$$


Coming back to (4.11) we choose there $\xi=D \boldsymbol{u}_{0}(x, t)$ (for arbitrarily fixed $(x, t) \in$ $\left.Q_{T}\right)$. Comparing the resulting equation with (4.12) and using the density of $\mathcal{D}_{A P}\left(\mathbb{R}_{\tau}\right) \otimes \mathcal{V}_{\text {div }}$ in $\mathcal{B}_{A P}^{p}\left(\mathbb{R}_{\tau} ; \mathcal{B}_{\text {div }}^{1, p}\right)$ we get by the uniqueness of the solution of (4.11) that $\boldsymbol{u}_{1}=\boldsymbol{\pi}\left(D \boldsymbol{u}_{0}\right)$, where $\boldsymbol{\pi}\left(D \boldsymbol{u}_{0}\right)$ stands for the function $(x, t) \mapsto \boldsymbol{\pi}\left(D \boldsymbol{u}_{0}(x, t)\right)$ considered as acting from $Q_{T}$ into $\mathcal{W}_{\text {div }}$. This shows the uniqueness of the solution of (4.9) and also allows us to conclude that $\boldsymbol{u}_{1}$ lies in $L^{p}\left(Q_{T} ; \mathcal{W}_{\text {div }}\right)$ so that $\left(\boldsymbol{u}_{0}, \boldsymbol{u}_{1}\right) \in \mathbb{F}_{0}^{1, p}$. This concludes the proof of Theorem 6 .

In order to get the macroscopic homogenized problem, we set, for $\xi \in \mathbb{R}^{N \times N}$,

$$
M(\xi)=\int_{\mathcal{K}} \widehat{b}|\xi+\partial \widehat{\boldsymbol{\pi}}(\xi)|^{p-2}(\xi+\partial \widehat{\boldsymbol{\pi}}(\xi)) d \beta
$$

and

$$
\mathrm{m} \xi=\int_{\mathcal{K}} \widehat{a}(\xi+\partial \widehat{\boldsymbol{\pi}}(\xi)) d \beta .
$$

Next, consider the following problem: Find $\left(\boldsymbol{u}_{0}, q_{0}\right)$ solution to

$$
\left\{\begin{array}{l}
\left.\mathfrak{M}_{y}(\rho) \frac{\partial \boldsymbol{u}_{0}}{\partial t}-\operatorname{div}\left(\mathbf{m} D \boldsymbol{u}_{0}\right)+M\left(D \boldsymbol{u}_{0}\right)\right)+B\left(\boldsymbol{u}_{0}\right)+\nabla q_{0}=\mathfrak{M}\left(\rho f\left(\cdot, \boldsymbol{u}_{0}\right)\right) \\
\operatorname{div} \boldsymbol{u}_{0}=0 \text { in } Q_{T} \\
\boldsymbol{u}_{0}=0 \text { on } \partial Q \times(0, T) \\
\boldsymbol{u}_{0}(x, 0)=\boldsymbol{u}^{0}(x) \text { in } Q
\end{array}\right.
$$

Problem (4.13) is the macroscopic homogenized problem. In contrast to (4.4) it only involves the macroscopic limit $\left(\boldsymbol{u}_{0}, q_{0}\right)$ of the sequence $\left(\boldsymbol{u}_{\varepsilon}, q_{\varepsilon}\right)$ of solutions to (2.1). Thus it describes the macroscopic behavior of the above-mentioned sequence as the heterogeneities go to zero.

Returning to Problem (4.13) we know that $\mathfrak{M}_{y}(\rho)$ is a positive constant (see Assumption (A5) at the beginning of this section). Moreover one may easily check that the functional $\boldsymbol{u} \mapsto \mathfrak{M}(\rho f(\cdot, \boldsymbol{u}))$ maps $\mathbb{H}$ into $L^{2}\left(Q_{T}\right)^{N}$, and is Lipschitz continuous (this is due to the properties of $f$ and $\rho$ ). Thus, assuming the existence of the velocity $\boldsymbol{u}_{0}$ in the above problem, it comes by [35, Proposition 5] that the pressure $q_{0}$ will exist. We can hence argue as in the proof of Theorem 1 to get the existence of a solution to (4.13). We may therefore state the macroscopic homogenization result.

Theorem 7. Assume that (A1)-(A5) hold. Let $1+\frac{2 N}{N+2} \leq p<\infty$. For each $\varepsilon>0$ let $\left(\boldsymbol{u}_{\varepsilon}, q_{\varepsilon}\right)$ be a solution of (2.1). Then there exists a subsequence of $\left(\boldsymbol{u}_{\varepsilon}, q_{\varepsilon}\right)_{\varepsilon>0}$ (not relabeled) which converges strongly in $L^{2}\left(Q_{T}\right)^{N}$ (with respect to the first component $\left.\boldsymbol{u}_{\varepsilon}\right)$ and weakly in $L^{p^{\prime}}\left(Q_{T}\right)$ (with respect to $\left.q_{\varepsilon}\right)$ to the solution of (4.13). Moreover any limit point in $L^{2}\left(Q_{T}\right)^{N} \times L^{p^{\prime}}\left(Q_{T}\right)$ (in the above sense) of $\left(\boldsymbol{u}_{\varepsilon}, q_{\varepsilon}\right)_{\varepsilon>0}$ is a solution to Problem (4.13).

Proof. Considering (4.8), by a density argument, this equation still holds for $\boldsymbol{\Phi}$ in $L^{p}\left(0, T ; W_{0}^{1, p}(Q)^{N}\right) \times L^{p}\left(Q_{T} ; \mathcal{W}_{\text {div }}\right)$. So substituting $\boldsymbol{u}_{1}=\boldsymbol{\pi}\left(D \boldsymbol{u}_{0}\right)$ in (4.8) and choosing the particular $\boldsymbol{\Phi}=\left(\boldsymbol{\psi}_{0}, \boldsymbol{\psi}_{1}\right)$ with $\boldsymbol{\psi}_{1}=0$ and $\boldsymbol{\psi}_{0} \in \mathcal{C}_{0}^{\infty}\left(Q_{T}\right)^{N}$, we end up (using some obvious computations) with the result.

\section{REFERENCES}

[1] R.A. Adams, Sobolev spaces, Academic Press, New York, 1975.

[2] H. Bellout, F. Bloom, J. Nečas, Young measure-valued solutions for non-Newtonian incompressible fluids, Commun. Partial Diff. Equ. 19 (1994) 1763-1803.

[3] A.S. Besicovitch, Almost periodic functions, Cambridge, Dover Publications, 1954. 
[4] J. Blot, Oscillations presque périodiques forcées d'équations d'Euler-Lagrange, Bull. Soc. Math. France 122 (1994) 285-304.

[5] H. Bohr, Almost periodic functions, Chelsea, New York, 1947.

[6] N. Bourbaki, Topologie générale, Chap. 1-4, Hermann, Paris, 1971.

[7] A. Bourgeat, A. Mikelić, S. Wright, Stochastic two-scale convergence in the mean and applications, J. Reine Angew. Math. 456 (1994) 19-51.

[8] T. Caraballo, J. Real, Navier-Stokes equations with delays, Proc. R. Soc. Lond. A. 457 (2001) 2441-2453.

[9] T. Caraballo, J. Real, A.M. Márquez, Three-dimensional system of globally modified NavierStokes equations with delay, Internat. J. Bifur. Chaos Appl. Sci. Engrg. 20 (2010) 2869-2883.

[10] H. Cheng, Resonance and generalized Navier-Stokes equations, Syst. Sci. Math. Sci. 13 (2000) $250-259$.

[11] C. Chou, Weakly almost periodic functions and Fourier-Stieltjes algebras of locally compact groups, Trans. Amer. Math. Soc. 274 (1982) 141-157.

[12] H. Douanla, Two-Scale convergence of elliptic spectral problems with indefinite density function in perforated domains, To appear in Asymtotic Analysis. (arXiv:1106.3907v1, 2011).

[13] H. Douanla, N. Svanstedt, Reiterated homogenization of linear eigenvalue problems in multiscale perforated domains beyond the periodic setting, Commun. Math. Anal. 11 (2011) 61-93.

[14] H. Douanla, N. Svanstedt, Homogenization of a nonlinear elliptic problem with large nonlinear potential, Appl. Anal. 91 (2012) 1205-1218.

[15] N. Dunford, J.T. Schwartz, Linear operators, Parts I and II, Interscience Publishers, Inc., New York, 1958, 1963.

[16] Y. Efendiev, A. Pankov, Homogenization of nonlinear random parabolic operators, Adv. Differ. Equ. 10 (2005) 1235-1260.

[17] C.H. Fefferman, Existence and smoothness of the Navier-Stokes equations, Clay Mathematics Institute, Preprint.

[18] Q. Du, M.D. Gunzburger, Analysis of a Ladyzhenskaya model for incompressible viscous flow, J. Math. Anal. Appl. 155 (1991) 21-45.

[19] V.V. Jikov, S.M. Kozlov, O.A. Oleinik, Homogenization of differential operators and integral functionals, Springer-Verlag, Berlin, 1994.

[20] O. Ladyzhenskaya, New equations for the description of the viscous incompressible fluids and solvability in the large of the boundary value problems for them, in "Boundary value problems of mathematical physics V", Amer. Math. Soc., Providence, RI, 1970.

[21] B.M. Levitan, Almost-Periodic Functions [in Russian], Gostekhizdat, Moscow, 1953.

[22] J.L. Lions, Quelques méthodes de résolution des problèmes aux limites non linéaires, Dunod, Paris, 1969.

[23] G. Nguetseng, Homogenization structures and applications I, Z. Anal. Anwen. 22 (2003) $73-107$.

[24] G. Nguetseng, M. Sango, J.L. Woukeng, Reiterated ergodic algebras and applications, Commun. Math. Phys. 300 (2010) 835-876.

[25] G. Nguetseng, J.L. Woukeng, Deterministic homogenization of parabolic monotone operators with time dependent coefficients, Electron. J. Differ. Equ. 2004 (2004) 1-23.

[26] G. Nguetseng, J.L. Woukeng, $\Sigma$-convergence of nonlinear parabolic operators, Nonlin. Anal. TMA 66 (2007) 968-1004.

[27] A. Novotný, I. Straškraba, Introduction to the mathematical theory of compressible flow, Oxford Univ. Press, 2004.

[28] M. Sango, N. Svanstedt, J.L. Woukeng, Generalized Besicovitch spaces and application to deterministic homogenization, Nonlin. Anal. TMA 74 (2011) 351-379.

[29] P.A. Razafimandimby, M. Sango, J.L. Woukeng, Homogenization of a stochastic nonlinear reaction-diffusion equation with a large reaction term: The almost periodic framework J. Math. Anal. Appl. 394 (2012) 186-212.

[30] M. Sango, J.L. Woukeng, Stochastic two-scale convergence of an integral functional, Asymptotic Anal. 73 (2011) 97-123.

[31] M. Sango, J.L. Woukeng, Stochastic sigma-convergence and applications, Dynamics of PDEs 8 (2011) 261-310.

[32] R.E. Showalter, Monotone operators in Banach spaces and nonlinear partial differential equations, in Mathematical Surveys and Monographs, Vol 48, AMS Providence, 1997. 
[33] L. Schwartz, Théorie des distributions, Hermann, Paris, 1966.

[34] J. Simon, On the identification $H=H^{\prime}$ in the Lions theorem and a related inaccuracy, Ric. Mat. 59 (2010) 245-255.

[35] J. Simon, On the existence of the pressure for solutions of the variational Navier-Stokes equations, J. Math. Fluid Mech. 1 (1999) 225-234.

[36] J.S. Smagorinsky, General circulation model of the atmosphere, Mon. Weather Rev. 91 (1963) 99-164.

[37] N. Svanstedt, J.L. Woukeng, Periodic homogenization of strongly nonlinear reaction-diffusion equations with large reaction terms, Applicable Analysis 2012, 1-22, iFirst.

[38] R. Temam, Navier-Stokes equations: Theory and numerical analysis, North-Holland, Amsterdam, 1984.

[39] K. Vo-Khac, Etude des fonctions quasi-stationnaires et de leurs applications aux équations différentielles opérationnelles, Mémoire Soc. Math. France 6 (1966) 3-175.

[40] S. Wright, Time-dependent Stokes flow through a randomly perforated porous medium, Asympt. Anal. 23 (2000) 257-272.

[41] J.L. Woukeng, $\Sigma$-convergence and reiterated homogenization of nonlinear parabolic operators, Commun. Pure Appl. Anal. 9 (2010) 1753-1789.

H. Douanla, Department of Mathematical Sciences, Chalmers University of TechNOLOGy, Gothenburg, SE-41296, Sweden

E-mail address: douanla@chalmers.se

J.L. Woukeng, Department of Mathematics and Computer Science, University of Dschang, P.O. Box 67, Dschang, Cameroon

E-mail address: jwoukeng@yahoo.fr 